\title{
A DTN-Based RFID Protocol
}

by

\author{
Xin Deng \\ A Thesis submitted to \\ the Faculty of Graduate Studies and Research \\ in partial fulfilment of \\ the requirements for the degree of \\ Master of Computer Science
}

Ottawa-Carleton Institute for

Computer Science

School of Computer Science
Carleton University
Ottawa, Ontario, Canada

Fall 2012

Copyright (C) 2012 - Xin Deng 
Library and Archives

Canada

Published Heritage

Branch

395 Wellington Street

Ottawa ON K1A ON4

Canada
Bibliothèque et

Archives Canada

Direction du

Patrimoine de l'édition

395 , rue Wellington

Ottawa ON K1A ON4

Canada
Your file Votre référence

ISBN: 978-0-494-94277-2

Our file Notre référence

ISBN: $978-0-494-94277-2$
NOTICE:

The author has granted a nonexclusive license allowing Library and Archives Canada to reproduce, publish, archive, preserve, conserve, communicate to the public by telecommunication or on the Internet, loan, distrbute and sell theses worldwide, for commercial or noncommercial purposes, in microform, paper, electronic and/or any other formats.

The author retains copyright ownership and moral rights in this thesis. Neither the thesis nor substantial extracts from it may be printed or otherwise reproduced without the author's permission.
AVIS:

L'auteur a accordé une licence non exclusive permettant à la Bibliothèque et Archives Canada de reproduire, publier, archiver, sauvegarder, conserver, transmettre au public par télécommunication ou par l'Internet, prêter, distribuer et vendre des thèses partout dans le monde, à des fins commerciales ou autres, sur support microforme, papier, électronique et/ou autres formats.

L'auteur conserve la propriété du droit d'auteur et des droits moraux qui protege cette thèse. $\mathrm{Ni}$ la thèse ni des extraits substantiels de celle-ci ne doivent être imprimés ou autrement reproduits sans son autorisation.
In compliance with the Canadian Privacy Act some supporting forms may have been removed from this thesis.

While these forms may be included in the document page count, their removal does not represent any loss of content from the thesis.
Conformément à la loi canadienne sur la protection de la vie privée, quelques formulaires secondaires ont été enlevés de cette thèse.

Bien que ces formulaires aient inclus dans la pagination, il n'y aura aucun contenu manquant. 


\section{Abstract}

In this thesis, we propose a lightweight Radio-frequency identification (RFID) mutual authentication protocol without permanent backend server connections. Only pseudo-random number generator $(P R N G)$ and $X O R$ operations are used in our protocol. Mutual authentication is achieved by exchanging random numbers drawn from the synchronized $P R N G$ functions of the readers and tags. A Delay Tolerant Network (DTN) is implemented by readers to communicate and synchronize their state. The performance is evaluated using the $O N E$ simulator. 


\section{Acknowledgments}

I would like to give special thanks to Michel Barbeau, professor at Carleton University

School of Computer Science, for his guidance and help. 


\section{Table of Contents}

Abstract

Acknowledgments $\quad$ iii

Table of Contents $\quad$ iv

Glossary $\quad$ v

1 Introduction 1

1.1 Background ....................... 1

1.1.1 Radio-frequency identification (RFID) technology . . . . . . . . . 1

1.2 Problem description . . . . . . . . . . . . . . . . . 2

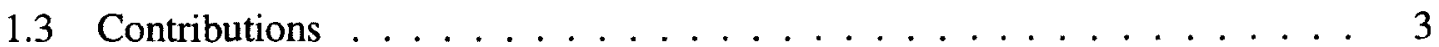

1.4 Document structure ........................ 4

2 Recent RFID security studies $\quad 6$

2.1 The Gossamer protocol . . . . . . . . . . . . . . . . 7

2.2 The CD protocol . . . . . . . . . . . . . . 10

2.3 The Flyweight protocol . . . . . . . . . . . . . . . 12

3 The DTN based RFID protocol 15

3.1 Drawbacks of previous protocols ................ 15

3.1.1 Design requirement ................. 15 
3.2 The DTN based RFID protocol $\ldots \ldots \ldots \ldots \ldots \ldots$

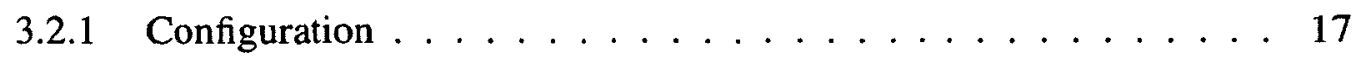

3.2 .2 Communication . . . . . . . . . . . . . . . . . 19

3.2.3 Resynchronization . . . . . . . . . . . . . 23

3.2 .4 Collision resolution . . . . . . . . . . . . . 25

4 Analysis and delay tolerant network 28

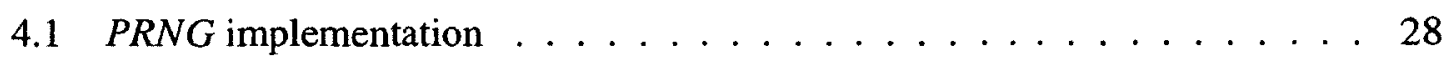

4.2 Compatibility analysis . . . . . . . . . . . . . . . . . . . 29

4.2.1 Protocol compatibility . . . . . . . . . . . . . . . . . . 29

4.2.2 Data compatibility . . . . . . . . . . . . 31

4.2.3 Data deployment . . . . . . . . . . . . . . . 32

4.3 Security analysis $\ldots \ldots \ldots \ldots \ldots \ldots \ldots \ldots \ldots$

4.3.1 Mutual verification . . . . . . . . . . . . . . 34

4.3.2 Data confidentiality . . . . . . . . . . . . . . . . 34

4.3 .3 Tag anonymity and untraceability $\ldots \ldots \ldots \ldots . \ldots 34$

4.3.4 Forward security $\ldots \ldots \ldots \ldots \ldots \ldots \ldots \ldots$

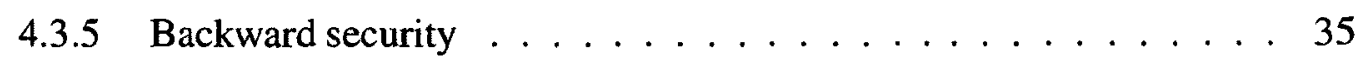

4.4 Delay tolerant network . . . . . . . . . . . . . . 35

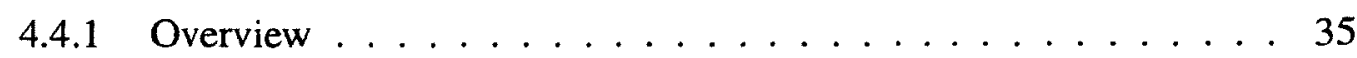

4.4.2 Delay-tolerant networking . . . . . . . . . . . 36

4.4 .3 DTN message routing $\ldots \ldots \ldots \ldots \ldots$

4.4.4 DTN and the DTN based RFID protocol . . . . . . . . . . 37

5 Simulation $\quad 38$

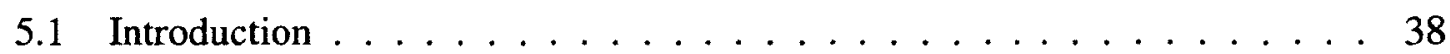

5.2 Scenario . . . . . . . . . . . . . . . . . . 39

5.3 Performance analysis $\ldots \ldots \ldots \ldots \ldots \ldots \ldots \ldots$ 
5.4 Simulation conclusion $\ldots \ldots \ldots \ldots$. . . . . . . . . . 49

6 Conclusion $\quad 50$

6.1 The DTN based RFID protocol . . . . . . . . . . . . . . . 50

6.2 Performance comparison .................... 51

6.2.1 Computation cost ................. 51

6.2 .2 Communication cost ................ 53

6.2 .3 Storage cost ................... 53

List of References $\quad 54$ 


\section{List of Tables}

1 Tag local constants and variables. . . . . . . . . . . . . 17

2 Reader local constants and variables of one tag. . . . . . . . . 18

3 Constant and variable definitions. . . . . . . . . . . . 18

4 FLAG values for different scenarios. . . . . . . . . . . . . . . . 19

5 The random number sequence generated from seed . . . . . . . . . . . 20

6 The random number sequence generated from seed s $^{\prime} \ldots \ldots$. . . . . . . 20

7 Inventory commands (they are all mandatory) . . . . . . . . . . . . . 29

8 Performance comparison. . . . . . . . . . . . . . 52 


\section{List of Figures}

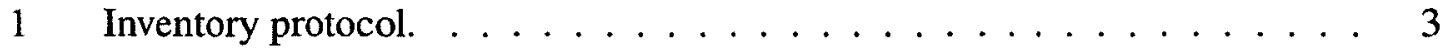

2 The Gossamer protocol. . . . . . . . . . . . . . . . . 8

3 The CD protocol. . . . . . . . . . . . . . . . . 11

4 A synchronized PRNG stream. . . . . . . . . . . . . . . 12

5 A de-synchronized PRNG stream. . . . . . . . . . . . . 12

6 The synchronized Flyweight protocol. . . . . . . . . . . . . . 13

7 The resynchronized Flyweight protocol. . . . . . . . . . . . . 14

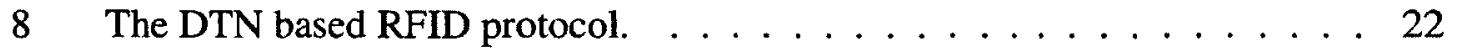

9 The DTN based RFID protocol resynchronization strategy. . . . . . . . . 24

10 The DTN based RFID protocol collision resolution strategy. . . . . . 26

11 The EPCglobal standard inventory protocol. . . . . . . . . . . 30

12 The DTN based RFID protocol. . . . . . . . . . . . . . 31

13 The map of a supermarket. . . . . . . . . . . . . . . . . . 39

14 The route map. . . . . . . . . . . . . . . . . 40

$15100 \%$ message generation - The total number of generated messages with the $90 \%$ confidence interval. . . . . . . . . . . . . . . 42

$1650 \%$ message generation - The total number of generated messages with the $90 \%$ confidence interval. . . . . . . . . . . . . . . . . 42

$17100 \%$ message generation - Message Coverage Rate with the $90 \%$ confidence interval. . . . . . . . . . . . . . . . . . . . . 44 43 
$1850 \%$ message generation - Message Coverage Rate with the $90 \%$ confidence interval. . . . . . . . . . . . . . . . . . . . . . . . . . 444

$19100 \%$ message generation - Message Update Success Rate with the 90\% confidence interval. . . . . . . . . . . . . . . . . . . . . . . . . 44

$2050 \%$ message generation - Message Update Success Rate with the $90 \%$ confidence interval. . . . . . . . . . . . . . . . . 45

21 The map of a warehouse. . . . . . . . . . . . . 46

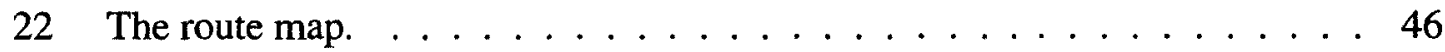

23 The total number of generated messages with the $90 \%$ confidence interval. . 47

24 Message Coverage Rate with the $90 \%$ confidence interval. . . . . . . . . 48

25 Message Update Success Rate with the $90 \%$ confidence interval. . . . . . . 48 


\section{Glossary}

\begin{tabular}{|c|c|}
\hline ACK & Acknowledgment \\
\hline $\mathrm{CRC}$ & Cyclic Redundancy Check \\
\hline EPC & Electronic Product Code \\
\hline $\mathrm{EPC} \mathrm{C1G2}$ & EPC Class 1 Gen2 Standard \\
\hline EPC HF C1G2 & EPC High Frequency Class 1 Generation 2 Standard \\
\hline EPC UHF C1G2 & EPC Ultra High Frequency Class 1 Generation 2 Standard \\
\hline HF & High Frequency \\
\hline ID & Identity \\
\hline IDS & Index-pseudonym Number \\
\hline LF & Low Frequency \\
\hline MDID & Tag Mask Designer Identifier \\
\hline NAK & Negative Acknowledgment \\
\hline ONE & Opportunistic Network Environment \\
\hline PRNG & Pseudo-random Number Generator \\
\hline
\end{tabular}




\begin{tabular}{|c|c|}
\hline RD & Reader Identity \\
\hline RFID & Radio-frequency Identification \\
\hline RN16 & 16-bit Random Number \\
\hline RND16 & 16-bit Random Number \\
\hline RND & Random Number \\
\hline seed $_{c u r}$ & Current PRNG Seed \\
\hline seed $_{\text {pre }}$ & Previous PRNG Seed \\
\hline seed $_{t e m p}$ & Temporary $P R N G$ Seed \\
\hline TD & Tag Identity \\
\hline TDS & Tag Pseudorandom Identity Number \\
\hline $\operatorname{TDS}_{c u r}$ & Current Tag Pseudorandom Identity Number \\
\hline $\mathrm{TDS}_{\text {pre }}$ & Previous Tag Pseudorandom Identity Number \\
\hline TID & Tag Identification \\
\hline TTL & Message Time To Live \\
\hline UHF & Ultra High Frequency \\
\hline URI & Uniform Resource Identifier \\
\hline XTID & Extended Tag Identification \\
\hline
\end{tabular}




\section{Chapter 1}

\section{Introduction}

\subsection{Background}

\subsubsection{Radio-frequency identification (RFID) technology}

The RFID (Radio-Frequency Identification) technology is widely used in daily transactions. It is slowly replacing the use of barcodes [4]. The implementation of a RFID tag consists of a processor, memory and a transceiver. It can be powered by a reader or use its own batteries. Each RFID tag has an Electronic Product Code (EPC). The EPC can be extracted by a portable reader unit.

Currently, there are three radio spectrum segments utilized by RFID: the Low Frequency $(L F)$ range (124 to $135 \mathrm{KHz}$ ), the High Frequency $(H F)$ range $(13.56 \mathrm{MHz})$ and the Ultra High Frequency $(U H F)$ range $(868 \mathrm{MHz}$ in Europe, $915 \mathrm{MHz}$ in North America or $950 \mathrm{MHz}$ in Japan) [2]. Chien classifies RFID tags into three types, based on supported operations: fullfledged, lightweight and ultralight [7]. Fullfledged tags are capable of conducting conventional cryptography. They support random number generators and hash functions. Fullfledged tags are high-cost. It means that they require more computation and memory resources to function. Lightweight tags implement random number generators and Cyclic Redundancy Code $(C R C)$ checksum calculation. Ultralight tags only support 
bitwise calculation operations like $X O R, A N D$ and $O R$. Lightweight tags and ultralight tags are low-cost. It means that they require less computation and memory resources. It is a big challenge to secure low-cost tags due to their restricted capabilities and widely distributed use in daily activities.

The EPCglobal standard [15] promotes the implementation of the RFID technology. It supports lightweight tags. It specifies an on-chip 16-bit Pseudo Random Number Generator $(P R N G)$ and a 16-bit Cyclic Redundancy Code calculator. EPCglobal [10] has defined a detailed standard architecture for system interoperability. This architecture has three parts: EPC Data Exchange standards, Data Exchange Infrastructure for Data Capture and Physical Object Exchange standards. Recently, the EPC Physical Object Exchange standard has been heavily studied due to the lack of a mutual verification scheme in the inventory protocol. The EPC Physical Object Exchange standard enables participant mutual identification. They define two interface standards: EPC UHF tag protocol [11] and EPC HF protocol [12]. They also define two data standards: tag data standard [13] and tag data translation standard [14]. The interface standards define detail commands that a reader can use to connect with tags. The tag data standards specify tag memory deployment and tag functionalities (e.g., $P R N G$ ). In this thesis, modifications are made to the inventory protocol in accordance with the requirements from the tag data standard and tag data translation standard.

\subsection{Problem description}

The inventory protocol is part of the interface standards. It specifies the start of a communication between a reader and a tag and helps the reader to identify a tag. Because of the absence of mutual verification in the inventory protocol, tags can be read without reader identity verification. The inventory protocol is presented in Figure 1. 


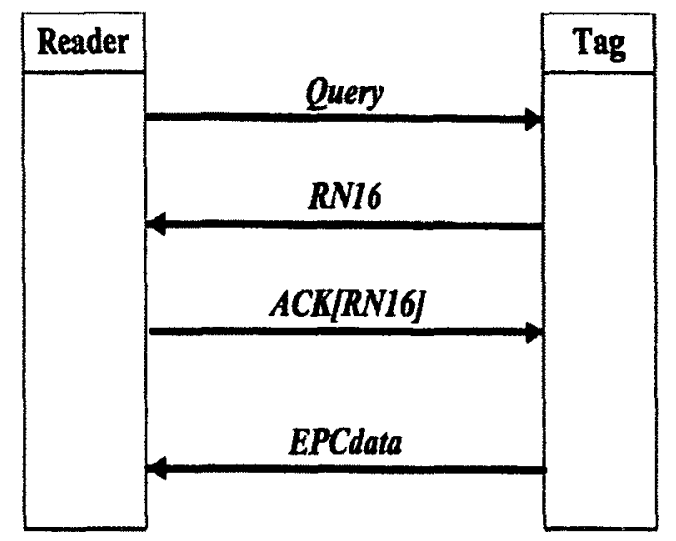

Figure 1: Inventory protocol.

Within the inventory protocol, a reader sends a query to a tag. The tag replies with a 16bit random number: $R N 16$. A tag accepts a reader when it receives the acknowledgement of the $R N 16$ from the reader. It sends back its identification $E P C$ dat $a$ back to the reader. Thus, as long as a reader is capable of sending a query and an acknowledgement of a random number, it can read any tag. Moreover, as long as a tag is equipped with a 16-bit random number generator, and can receive the query command, it can start a communication with a reader. On the other hand, adversaries can read and write a reader or forge a tag's identity and provide information to a reader on behalf the real tag. There is no mutual verification in the inventory protocol. If the tag is used as a chip on a credit card, then important personal information can be leaked to the adversary. Adversaries can also use a fake chip to complete transactions on behalf of a real credit card. Mutual verification is needed for the inventory protocol.

\subsection{Contributions}

Several new protocols have been proposed to solve the lack of mutual authentication in the EPCglobal inventory protocol defined in [11] and [12]. In Chapter 3, we propose a new 
mutual verification protocol - the DTN based RFID protocol. In the DTN based RFID protocol, readers and tags keep a synchronized $P R N G$ state, which includes the same $P R N G$ function and same $P R N G$ seed for mutual verification. Each tag is assigned a pseudonym number. It is used to communicate with the reader instead of using the its real identity. Both the $P R N G$ seed and tag's pseudonym number are updated after every successful inventory. The DTN based RFID protocol enables RFID tags and readers to verify each other. It helps RFID tags to hide their own real identity. It achieves untraceability, mutual verification and forward and backward security for lightweight RFID tags. If there is more than one reader working with a set of tags, reader states need to be synchronized using a permanent connection with a backend server or a network. We study two scenarios where the RFID technology is combined with Delay Tolerant Networks (DTNs) to avoid permanent connections with backend servers. All our works follow the requirement of the EPCglobal interface standard. The DTN based RFID protocol is fully compatible with the current EPCglobal standard.

\subsection{Document structure}

In this thesis, previous research is reviewed and summarized. The DTN based RFID protocol and two related strategies are introduced. The security of the protocol and its compatibility with the EPCglobal standards are discussed. A simulation is conducted to evaluate RFID readers' performance in the DTN. The following is the structure of this thesis.

Chapter 2, Recent RFID security studies. This chapter discusses three previous research works to enhance the EPCglobal inventory protocol security.

Chapter 3, The DTN based RFID protocol. This chapter presents our proposed protocol and strategies for handling a de-synchronization situation and a collision situation. 
Chapter 4, Analysis and delay tolerant network. This chapter discusses the security of the proposed protocol and its compatibility with the EPCglobal standard. At the end of this chapter, the concept of DTN and the strategy RFID readers use to communicate in a DTN are introduced.

Chapter 5, Simulation. Simulation is conducted in this chapter to evaluate RFID readers performance in two scenarios.

Chapter 6, Conclusion. This chapter summarizes the overall work and compares it with two previous proposed protocols. 


\section{Chapter 2}

\section{Recent RFID security studies}

EPCglobal standards aim at improving the RFID tag operationalability and balancing its cost and functionality. Basic reliability is guaranteed by the use of $P R N G$ and $C R C$ functions. Based on their standards, a reader needs to conduct three different protocol sessions to communicate with a tag: select, inventory, access. In the select session, reader selects a group of tags. The inventory session helps the reader to identify one tag from the selected tag group. The reader starts to update the chosen tag in the access session. In the inventory session, the RFID tag sends its identification to the reader for further communications. No mutual verification in the inventory session leads to the exposure of a tag identity. It is vulnerable to tag identity theft and reader impersonation.

Studies on the EPCglobal inventory protocol can be roughly divided into two categories. In the first category, new protocols use private and public keys with hash functions for mutual verification. Theoretically, they prove that hash functions can secure the EPCglobal inventory protocol. But, low-cost RFID tags do not support hash functions. The second category of studies focuses on using the current EPCglobal standard to design lightweight RFID protocols. Among the second category, there are two branches of popular protocols. One is the family of ultralight weight protocols: the first is $M^{2} A P$ [26], followed by EMAP [24] and LMAP [25]. This branch of protocols do not expose any tag or reader information in the environment. They completely follow the EPCglobal standard 
by using bitwise operations: $O R, A N D$ and $X O R$. The reader identifies tags by using tag pseudonym numbers instead of their own real identities. In 2009, authors of those protocols proposed a new one called Gossamer [28]. This new ultralight protocol has some ideas similar with the ones of the SASI protocol [7]. They both store the previous inventory verification information to recover from de-synchronization between a reader and a tag.

\subsection{The Gossamer protocol}

There are three groups of participants in the Gossamer Protocol [28]: the server, readers and tags. Readers are used to communicate with tags while the server supports all calculations and verifications. Instead of using a tag's real identity, an index-pseudonym number IDS is used for each tag. The IDS is updated after every successful interrogation. The tag real identification is kept the same. Tags and the server shares two private keys: $k_{1}, k_{2}$ and two security random number: $n_{1}, n_{2}$. Both the server and tags keep ID,IDS, $k_{1}, k_{2}$, $n_{1}$ and $n_{2}$ locally. Every time a reader receives an IDS, it communicates with the server. The server matches the IDS in the database and returns the corresponding $k_{1}, k_{2}, n_{1}$ and $n_{2}$ to the reader. The Gossamer protocol uses not only the EPCglobal standard defined operations: bitwise $X O R$, bitwise $A N D$, bitwise $O R$ but also new functions: $R O T(x, y)$ and $\operatorname{MIX} B I T S(x, y)$. These two new functions are used to calculate verification messages and update local variables. Function $R O T(x, y)$ is defined to conduct a circular shift for the bit sequence $x$, (y mod 96) positions to the left. Function MIXBIX $(x, y)$ uses bitwise right shift $(>>)$ and additions to compute an output $Z$ :

$$
\begin{aligned}
& Z=x ; \\
& \text { for }(i=0 ; i<32 ; i++) \\
& Z=(Z>>1)+Z+Z+Y ;
\end{aligned}
$$

The Gossamer protocol includes four steps and is illustrated in Figure 2: 


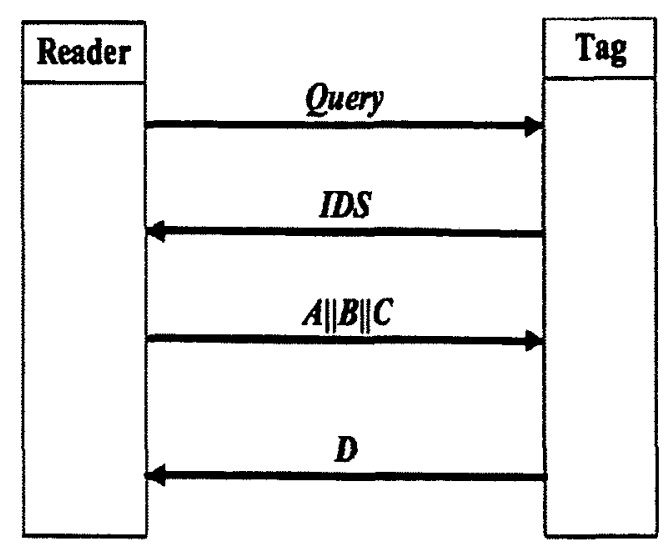

Figure 2: The Gossamer protocol.

Tag identification. A reader sends the query command to a tag. The tag replies with the $I D S$. The reader then sends the IDS to the server and gets the related $k_{1}, k_{2}$.

Mutual authentication. A reader generates $A, B$ and $C$ separately and sends them together to the tag. The function $R O T$ is used twice with five parameters to generate $A$ and $B$ according to the following equations 1 and 2 :

$$
\begin{aligned}
& A=R O T\left(R O T\left(I D S+k_{1}+\pi+n_{1}, k_{2}\right)+k_{1}, k_{1}\right) \\
& B=\operatorname{ROT}\left(\operatorname{ROT}\left(I D S+k_{2}+\pi+n_{2}, k_{1}\right)+k_{2}, k_{2}\right)
\end{aligned}
$$

Next, the function call $\operatorname{MIXBITS}\left(n_{1}, n_{2}\right)$ is used to generate $n_{3}$. The function call $\operatorname{MIXBIT}\left(n_{3}, n_{2}\right)$ is used to generate a new $n_{1}$ which is stored in the server as $n_{1}^{\prime}$. The $k_{1}$ and $k_{2}$ are updated by using the ROT function to $k_{1}^{\prime}$ and $k_{2}^{\prime}$ in equations 3 and 4:

$$
\begin{aligned}
& k_{1}^{\prime}=\operatorname{ROT}\left(\operatorname{ROT}\left(n_{2}+k_{1}+\pi+n_{3}, n_{2}\right) \oplus n_{3}, n_{1}\right) \oplus n_{3} \\
& k_{2}^{\prime}=\operatorname{ROT}\left(\operatorname{ROT}\left(n_{1}+k_{2}+\pi+n_{3}, n_{1}\right) \oplus n_{3}, n_{2}\right) \oplus n_{3}
\end{aligned}
$$


Then the $C$ is generated as:

$$
C=R O T\left(R O T\left(n_{3}+k_{1}^{\prime}+\pi+n_{1}^{\prime}, n_{3}\right)+k_{2}^{\prime} \oplus n_{1}^{\prime}, n_{2}\right) \oplus n_{1}^{\prime}
$$

The reader now concatenates $A, B, C$ together and sends the result to the tag. The tag extracts $n_{1}$ from $A$ and $n_{2}$ from $B$ by using its local $I D S, k_{1}, k_{2}$ to generate $C^{\prime}$ with the function 5. If $C=C^{\prime}$, the tag generates $D$ in the equation 6 :

$$
D=\operatorname{ROT}\left(\operatorname{ROT}\left(n_{2}+k_{2}^{\prime}+I D+n_{1}^{\prime}, n_{2}\right)+k_{1}^{\prime}+n_{1}^{\prime}, n_{3}\right)+n_{1}^{\prime}
$$

and the tag sends $D$ to the reader for verification.

Tag update and backend database update. A reader uses equation 6 to generate $D^{\prime}$ with its local variables. If $D=D^{\prime}$, then the backend server and tag update all the local parameters for the next verification. The function $\operatorname{MIXBITS}\left(n_{1}^{\prime}, n_{3}\right)$ is used to update $n_{2}$ and the IDS is updated in the equation 7 :

$$
I D S=R O T\left(R O T\left(n_{1}^{\prime}+k_{1}^{\prime}+I D S+n_{2}^{\prime}, n_{1}^{\prime}\right)+k_{2}^{\prime} \oplus n_{2}^{\prime}, n_{3}\right) \oplus n_{2}^{\prime}
$$

$k_{1}$ and $k_{2}$ are updated by using equations 3 and 4 again. In the backend database, previous $I D S, k_{1}$ and $k_{2}$ are stored to recover from an eventual de-synchronization attack. An adversary can block the last transmission from the tag to the reader, so that the tag can update its information while the reader does not update its local records due to the lack of the verification message from the tag. In order to recover from this de-synchronization attack, tags have to save both current IDS, $k_{1}, k_{2}$ and previous ones. If the database can not identify the current $I D S$, the reader requires a tag to send its IDS again. The tag replies with the previous IDS to resynchronize each other again. 
Progress and constrains. The Gossamer protocol achieves untraceability by using different $I D S$ for each inventory execution. The $I D S$ is updated after each successful inventory to look random to the environment. However, it uses two defined functions that are not supported by the current EPCglobal standard, i.e. ROT and MIXBIT. RFID tags need to be re-designed to implement the protocol. Moreover, an adversary can eavesdrop on the previous value of $A\|B\| C$ and the tag's previous $I D S$ to fake a de-synchronization scenario to communicate with a tag. In our proposed protocol, the IDS is also used to achieve untraceability. Only the EPCglobal standard compatible operations are used. And also, if a tag and a reader are de-synchronized, they need to recover from the de-synchronization and then verify each other again before starting a new communication.

\subsection{The CD protocol}

In the second branch, the $P R N G$ and $C R C$ functions are used to secure the inventory protocol. They are compatible with the EPCglobal standard. In the CD protocol [6], the CRC is used to encrypt the verification data. It does not need any permanent connection with a backend server but requires readers and tags to register to the server before they are put into use. Each tag shares three private parameters with the back-end server: a key $k$, a nonce $N$ and an unique identifier EPC. The CD protocol is shown in Figure 3.

Their inventory protocol includes three steps:

Tag identification. A reader inquiries a tag with three messages: a request: $M_{\text {req }}$, a $C R C$ function result $C R C(N \oplus R N D)$ and a random number $R N D$.

Reader verification. A tag verifies the $C R C(N \oplus R N D)$ by calculating the result with its local nonce $N$, the received $R N D$ and the $C R C$ function. If this $C R C$ result is verified, a tag generates a new random number $\left(R N D_{\text {new }}\right)$ and computes tag-verification information: 


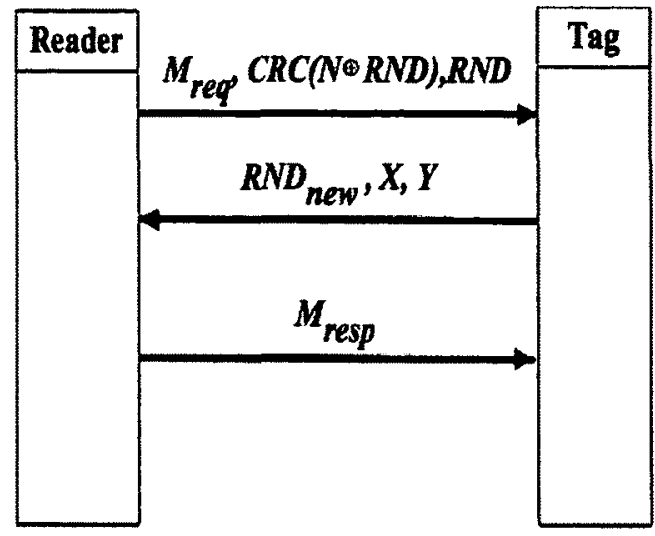

Figure 3: The CD protocol.

$X$ and $Y$ with its identification information $E P C$, local key $k$ and nonce $N$. Calculations are shown in equations 8 and 9. A tag sends $X, Y$ and $R N D_{n e w}$ to the reader.

$$
X=\left(k \oplus E P C \oplus R N D_{\text {new }}\right)
$$

and

$$
Y=C R C\left(R N D_{n e w} \oplus N \oplus X\right)
$$

Tag verification. A reader verifies the received $X$ and $Y$ by using equations 8 and 9 with its local variables and the $R N D_{n e w}$. If the tag is verified, the reader sends a response to the tag.

Progress and constrains. The use of the $C R C$ function for the verification is proven insecure. Indeed, an adversary can successfully respond a reader's query after the eavesdropping of one interrogation session between the reader and a tag [22]. Although the CD protocol is proven insecure, it is the first one to propose an inventory protocol without a permanent backend server connection. The DTN based RFID protocol includes two stages as well. Readers and tags are configured with private secure parameters first before they 
are put into use. Readers then use a DTN to communicate with each other, so that no permanent backend connection is needed.

\subsection{The Flyweight protocol}

In 2009, a new protocol called Flyweight [5] based on the use of the PRNG function was proposed. This protocol closely follows the EPCglobal standard and prevents leaking of any tags or reader identities during the interrogation. This protocol requires readers and tags to use a synchronized $P R N G$ state (same $P R N G$ algorithm and $P R N G$ seed) to generate the identical random number sequence. They then use different parts of the random number sequence for mutual verification. Similarly to the Gossamer protocol, a permanent server connection is needed.

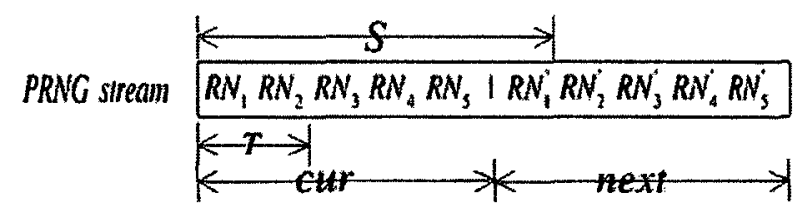

Figure 4: A synchronized PRNG stream.

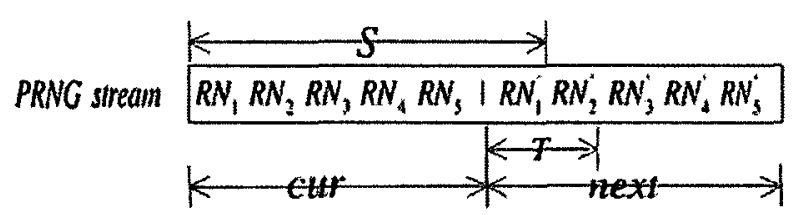

Figure 5: A de-synchronized PRNG stream.

The Flyweight protocol uses random number sequences generated by a $P R N G$ function with a 32-bit seed. Every random number sequence is divided into 5 random numbers: $\left(R N_{1}, R N_{2}, R N_{3}, R N_{4}, R N_{5}\right)$; each number is 16 bits long. As shown in Figures 4 and 5 , 
the $S$ stands for the server and the $T$ stands for the tag, there are two continuous random number sequences: the current five random numbers $\left(R N_{1}\right.$ to $\left.R N_{5}\right)$ and the next five random numbers $\left(R N_{1}^{\prime}\right.$ to $R N_{5}^{\prime}$ ) . A reader is connected with a database that always holds six numbers. They include the first random number of the current sequence $R N_{1}$ to the first number of the next sequence $R N_{1}^{\prime}$. A tag holds the current $R N_{1}, R N_{2}$ if it is synchronized with the reader (Figure 4) or holds the next $R N_{1}^{\prime}, R N_{2}^{\prime}$ if it is not (Figure 5). The de-synchronization occurs when a tag updates its random sequence to the next one while the reader still holds the current random sequence. If the reader and the tag are synchronized, $R N_{1}, R N_{2}, R N_{3}$ from the same sequence are then used for mutual verification as it is shown in Figure 6. If it is a de-synchronization situation, $R N_{1}^{\prime} R N_{2}^{\prime}, R N_{3}^{\prime}, R N_{4}^{\prime}, R N_{5}^{\prime}$ from next sequences are used for resynchronization and verification as it is shown in Figure 7.

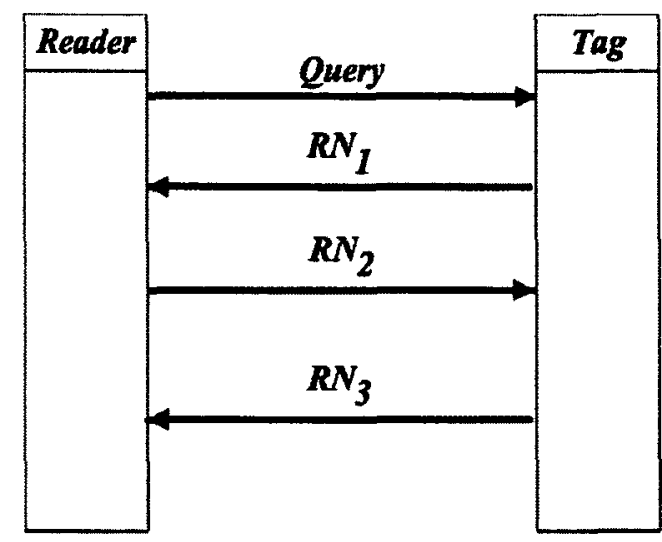

Figure 6: The synchronized Flyweight protocol.

A reader first sends a query to a tag, the tag then replies with $R N_{1}$. If the received $R N_{1}$ is the current $R N_{1}$ then $R N_{2}$ is replied to the tag. If the received $R N_{2}$ equals to the local $R N_{2}$, the tag generates the random sequence from $R N_{3}$ to $R N_{2}^{\prime}$. The tag replaces $R N_{1}$ and $R N_{2}$ with $R N_{1}^{\prime}$ and $R N_{2}^{\prime}$ in its memory. The tag sends the $R N_{3}$ back to the reader for verification. If the $R N_{3}$ is valid, the reader updates random number sequences and this mutual verification session is completed. 


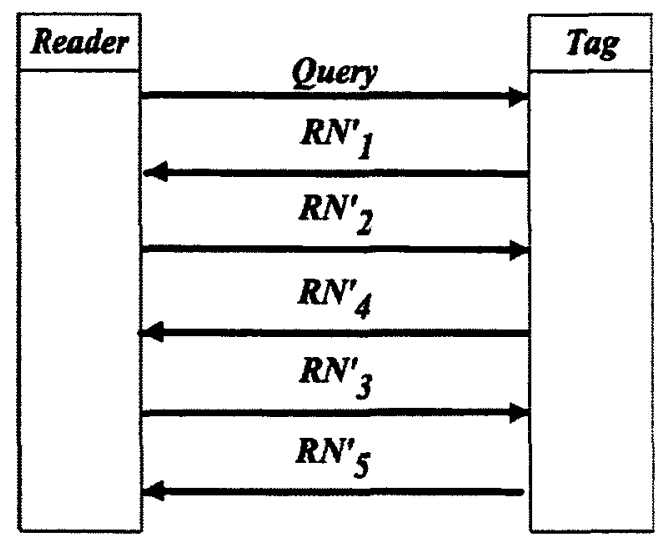

Figure 7: The resynchronized Flyweight protocol.

In Figure 7, if the reader and the tag are de-synchronized, the reader receives the next $R N_{1}^{\prime}$. The reader then updates its random number sequence and replies $R N_{2}^{\prime}$ with a flag to alert the de-synchronization. The tag then updates its random number sequence and replies with the $R N_{4}^{\prime}$ to the reader. To acknowledge that $R N_{4}^{\prime}$ has been received, the reader sends back $R N_{3}^{\prime}$ and updates its random number sequences but saves the old $R N_{5}^{\prime}$. The tag then receives $R N_{3}^{\prime}$, if it equals to the local one, the tag sends back $R N_{5}^{\prime}$. If the received $R N_{5}^{\prime}$ equals to the old $R N_{5}^{\prime}$ of the reader, then the tag is accepted. This process is shown in Figure 6. Overall, the tag updates its random sequence once, while the reader updates its local sequence twice.

Progress and constrains. With the use of the PRNG function, readers and tags keep less information for mutual verification compared to the Gossamer protocol. A PRNG seed needs to be updated for readers and tags. The function and the frequency to update the PRNG seed is not specified in their paper. The DTN based RFID protocol uses random number sequences for mutual verification, and after every successful inventory, the $P R N G$ seed is updated. 


\section{Chapter 3}

\section{The DTN based RFID protocol}

\subsection{Drawbacks of previous protocols}

The Gossamer protocol uses two functions: MIXBITS and ROT which are not supported by current lightweight RFID tags. The Flyweight protocol [5] implements a PRNG function for mutual verification, but the $P R N G$ seed update frequency and update function are not specified. Furthermore, the EPCglobal standard does not define connections with a backend database in the inventory protocol. Both the Gossamer and Flyweight protocols, however, require permanent backend server connections. The Gossamer protocol has to use a database to match the tag, generate verification messages and update private keys. A reader is required to connect with the sever all the time. In the Flyweight protocol, the server not only needs to process the data as the Gossamer protocol, but it also has to update the PRNG seed.

\subsubsection{Design requirement}

New designed RFID protocols should achieve compatibility, security and prevent permanent server connections. For the compatibility, the protocol must follow EPCglobal standards. There are four requirements for the protocol security. Firstly, both the tag and reader 
need to verify each other before conduct further communications. Secondly, adversaries can not link any messages to a tag based on previous ones, which they eavesdrop from the environment. All the tags are untraceable. Thirdly, Previous messages confidentiality is ensured, even if the tag's current local parameters are exposed to an adversary. And fourthly, to an adversary who has access to a tag's current local parameters, future messages keep confidential to the adversary after the tag updates its current parameters.

The DTN based RFID protocol is designed to provide mutual verification without permanent backend server connections. Particularly, it focuses on the following design requirements:

Compatibility. The DTN based RFID protocol only uses functions and operations that are supported by RFID lightweight tags including the $P R N G$ function and $X O R$ operation. The Flyweight protocol [5] and studies from [24], [26], [28] have shown that the PRNG function can be used in accordance with the requirements from the EPCglobal standard.

No server connections. In the DTN based RFID protocol, the initial set of verification parameters are configured within the readers and tags. A DTN is used by readers to exchange new information. Each reader in the DTN carries, stores and broadcasts new messages to other readers.

Security. The DTN based RFID protocol protocol is designed to meet mutual verification, untraceability, forward security, backward security.

\subsection{The DTN based RFID protocol}

The DTN based RFID protocol focuses on mutual verification without permanent backend server connections. It includes two stages: configuration and communication. During the configuration stage, readers and tags obtain the initial set of private secure parameters. In 
Table 1: Tag local constants and variables.

\begin{tabular}{l|l|l|l}
\hline$T D$ & $R D$ & $T D S$ & seed \\
\hline
\end{tabular}

the communication stage, the DTN based RFID protocol conducts mutual verification and uses a DTN to connect all readers.

\subsubsection{Configuration}

The configuration stage is conducted in a secure environment. A tag obtains its identity $T D$, its pseudonym number $T D S$, a reader identity $R D$ and an initial $P R N G$ function seed as it is shown Table 1.TD and $R D$ are constants. $T D$ is for tag identification and $R D$ is used to verify readers. TDS and seed are variables and updated after every successful inventory. A tag only has one record of the type of Table 1 . A reader needs to use the valid $R D$ to access it.

A reader acquires its identity $R D$ and a list of accessible tag information records. For each tag in the list, a reader stores its $T D$, previous session $T D S\left(T D S_{p r e}\right)$, current session TDS $\left(T D S_{c u r}\right)$, previous $P R N G$ seed $\left(\right.$ seed $\left._{\text {pre }}\right)$ and current $P R N G$ seed (seed cur $_{\text {) }}$ as it is shown in Table 2. In our proposed protocol, a list of tags is associated to one $R D$. Readers that share the same $R D$ have access to the same list of tags. In the configuration stage, no tag previous $P R N G$ seed $\left(\right.$ seed $\left._{\text {pre }}\right)$ and $T D S\left(T D S_{\text {pre }}\right)$ are available for readers. Table 2 details one tag information record of a reader's local tag list. Several RDs can be assigned to a reader to access correlated tags. $T D S_{\text {pre }}, T D S_{c u r}$, seed $_{\text {pre }}$ and seed cur $_{\text {che variables and }}$ are updated after a tag is read. The updated information is a new message. The message is sent to other readers to update their local record of the same tag. All parameters are defined in Table 3.

Readers and tags keep the same $P R N G$ state, which includes the same seed and $P R N G$ algorithm in order to generate an identical sequence of random bits. Different parts of the 
Table 2: Reader local constants and variables of one tag.

\begin{tabular}{l|l|l|l|l|l}
\hline$T D$ & $T D S_{\text {pre }}$ & $T D S_{\text {cur }}$ & seed $_{\text {pre }}$ & seed $_{c u r}$ & $R D$ \\
\hline \hline
\end{tabular}

Table 3: Constant and variable definitions.

\begin{tabular}{l|l}
\hline$T D$ & 32-bit tag identifier \\
\hline$R D$ & 32-bit reader identifier \\
\hline$T D S_{\text {pre }}$ & 16-bit previous pseudonym number of a tag \\
\hline$T D S_{\text {cur }}$ & 16-bit current pseudonym number of a tag \\
\hline seed $_{\text {pre }}$ & 16-bit previous $P R N G$ seed \\
\hline seed $_{\text {cur }}$ & 16-bit current $P R N G$ seed \\
\hline seed $^{\prime}$ & 16 -bit $P R N G$ seed \\
\hline$R N D 16$ & 16-bit random number \\
\hline$P R N G_{n: m}($ seed $)$ & $\begin{array}{l}\text { A random number that starts at bit } n \text { and finishes } \\
\text { at bit } m \text { in a random number sequence generated } \\
\text { by } P R N G \text { with a seed. }\end{array}$ \\
\hline \hline
\end{tabular}


Table 4: FLAG values for different scenarios.

\begin{tabular}{l|l|l}
\hline FLAG=0 & FLAG=1 & FLAG=2 \\
\hline synchronization & de-synchronization & collision \\
\hline \hline
\end{tabular}

random sequence are segregated into several random numbers. Each number starts at bit $\mathbf{n}$ and finishes at bit $\mathrm{m}$ is defined as $P R N G_{n: m}($ seed $)$.

A tag's $P R N G$ seed and TDS are updated every time after successful mutual verification, so that the verification information is different in every inventory. However, if an adversary blocks a certain transmission between the reader and tag, it can cause a desynchronized $P R N G$ state: The reader updates its local parameters while the tag still keeps the current one. This de-synchronization attack is discussed in detail in the sub-section 3.2.3. To solve this security issue, readers store previous verification information, including the previous $P R N G$ seed (seed $p$ re) and the tag's previous pseudonym number (TDS $S_{\text {pre }}$ ).

Another possible issue may occur after updating a TDS. Several tags can have the same $T D S$, which the reader cannot differentiate. The TDS collision problem is discussed in detail in the sub-section 3.2.4.

A FLAG parameter is used by the reader in the inventory protocol to inform a tag that a de-synchronization or a collision has occurred. The different values of the FLAG and their meanings are shown in Table 4.

\subsubsection{Communication}

\section{The usage of a random number sequence}

Two PRNG seeds are used in the DTN based RFID protocol: seed and seed'. The seed is originally obtained through configuration and the reader stores it as seed ${ }_{c u r}$. seed $^{\prime}$ is dynamically generated in every inventory session. Readers and tags update their local seed 
with the constant $R D$, the previous seed and a 32-bit random number after every successful mutual verification. A 16-bit number drawn from it is to prove that the tag holds the valid $R D$. The seed is generated with the constant $T D$, the current seed and a 32-bit random number. A 16-bit number drawn from it is to show the reader has access to the tag with the identity $T D$.

Table 5: The random number sequence generated from seed.

\begin{tabular}{l|l|l|l}
\hline PRNG $_{\text {0:15 }}($ seed $)$ & PRNG $_{16: 47}($ seed $)$ & PRNG $_{48: 79}($ seed $)$ & PRNG $_{80: 111}($ seed $)$ \\
\hline 16-bit number to & $\begin{array}{l}\text { 32-bit number for } \\
\text { seed update (First } \\
\text { prove a reader iden- } \\
\text { tity }\end{array}$ & $\begin{array}{l}\text { 32-bit number for are for TDS } \\
\text { update) }\end{array}$ & $\begin{array}{l}\text { seed' generation } \\
\text { resynchronization }\end{array}$ \\
\hline \hline
\end{tabular}

Table 6: The random number sequence generated from seed'.

\begin{tabular}{l|l|l}
\hline PRNG $_{0: 15}\left(\right.$ seed $\left.^{\prime}\right)$ & PRNG 16:31 $\left._{\text {seed }}\right)$ & $\begin{array}{l}\text { PRNG }_{32: 47}\left(\text { seed }^{\prime}\right) \\
\mathbf{P R N G}_{48: 63}\left(\text { seed }^{\prime}\right)\end{array}$ \\
\hline $\begin{array}{l}\text { 16-bit number to prove } \\
\text { a tag identity }\end{array}$ & $\begin{array}{l}\text { 16-bit number for TDS } \\
\text { update }\end{array}$ & $\begin{array}{l}\text { two 16-bit numbers for TDS } \\
\text { collision resolution }\end{array}$ \\
\hline \hline
\end{tabular}

Two random bit sequences are divided into four numbers each. The first random number sequence that is generated from the seed is shown in Table 5. It is 112 bit long. The first 16 bits of the sequence are used to prove a reader's identity. The 16 th bit to the 47 th bit in the sequence are used as a 32-bit random number to update the $s_{\text {eed }}$ cur for a reader or 
the seed for a tag. The first 16 bits of this 32-bit random number are used to update a tag's $T D S$. From the 48 th bit to the 79 th bit is another 32-bit random number to generate the seed $^{\prime}$. The last 32 bits are used as a random number to update the seed ${ }_{c u r}$ for a reader and seed for a tag when a de-synchronization occurs.

Table 6 details the second random number sequence that is generated from the seed'. it is 64 bit long. The first 16 bits of the random sequence are used to prove a tag's identity. From the 16 th bit to the 31 st bit of this sequence is a 16-bit random number to update a tag's $T D S$. The $32 \mathrm{nd}$ bit to the 47 th bit and the 48th bit to the 63rd are segregated into two 16-bit random numbers to resolve a collision.

\section{The DTN based RFID protocol}

This protocol includes four steps as it is shown in Figure 8.

Tag identification. First, a reader sends a Query to a tag as outlined in step 1. The tag replies its TDS back to the reader in step 2. If the reader and tag are synchronized with each other, the received $T D S$ is a $T D S_{c u r}$ in a reader's local record. The reader sets FLAG to 0 and backs up the seed cur $_{\text {as }}$ seed pre. A new seed $_{\text {cur }}$ is generated to draw a 16-bit random number ( $R N D 16)$ that is sent to the tag for reader verification. The seed $d^{\prime}$ is generated. The $T D S_{p r e}$ and $T D S_{c u r}$ are updated. The reader sends the 16-bit random number (RND16) and FLAG to the tag.

Reader verification. After the tag receives the RND16 and FLAG from the reader as

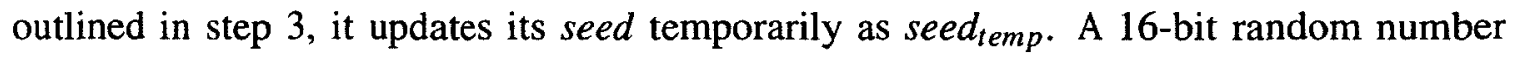
is generated from the temporary seed to verify the received $R N D 16$. Only if the received $R N D 16$ is verified, the reader is considered valid and the tag saves the temporary seed seed $_{\text {temp }}$ as seed and generate the seed'. The seed' then is used to draw a 16-bit random number $(R N D 16)$ to prove the tag identity to the reader. Before the tag sends the 16-bit 


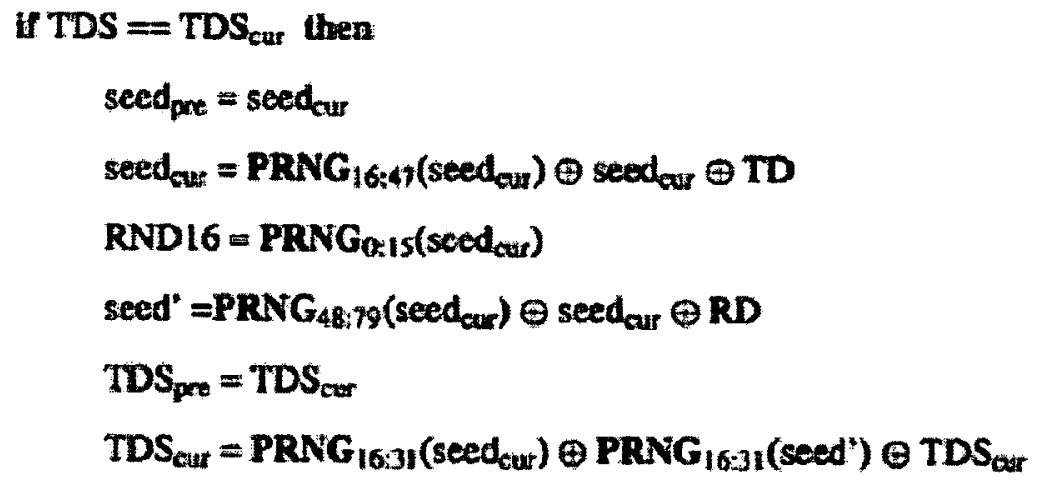

$$
\text { seed }_{\text {iemp }}=\operatorname{PRNG}_{16: \mathrm{T}}(\mathrm{secd}) \oplus \operatorname{seed} \oplus \mathrm{TD}
$$$$
\text { If } \text { PRNG }_{0.15}\left(\text { seed }_{\text {templp }}\right)=\mathrm{RND} 16 \text { then }
$$$$
\text { seed }=\text { seed }_{\text {remp }}
$$$$
\text { seed }^{\prime}=\text { PRNG }_{48: 79}(\text { seed }) \oplus \operatorname{seed} \oplus \mathbf{R D}
$$$$
\text { RND16 = PRNGo.1s(seed') }
$$$$
\text { TDS }=\text { PRNG }_{16331}(\text { seed }) \oplus \text { PRNG }_{16 \times 3 !}(\text { seed') } \oplus \text { TDS }
$$

\section{RND16}

if PRNGo:1s(seed') $=$ RND16

then Tag is valid.

Figure 8: The DTN based RFID protocol. 
random number back to the reader, the tag also updates its $T D S$.

Tag verification. In step 4, the reader receives the 16-bit random number (RND16) from the tag. It then uses the seed' to draw another 16-bit number. If it is equal to the received number, the tag is valid. The reader accepts the tag.

\subsubsection{Resynchronization}

Since the reader updates its local variables when the $T D S$ received from the tag is valid and the tag updates its local variables after it verified the reader, they do not update their local variables at the same time. An adversary can block the third transmission when the reader sends a 16-bit number $(R N D 16)$ to the tag. The reader updates its local variable but the tag remains in its current state and waits for a message from the reader. Thus, de-synchronization occurs.

The following strategy is used to resynchronize the reader and tag. It requires one interrogation to complete resynchronization. It also needs three steps as it is shown in Figure 9.

Tag and de-synchronization identification. In step 2, if a reader and a tag are desynchronized, the received $T D S$ equals the $T D S_{\text {pre }}$ of a reader's local record. The reader sets the FLAG to 1 to alert de-synchronization. The reader generates a temporary seed: seed $_{\text {temp }}$ to draw a 16-bit random number $(R N D 16)$ for the tag to verify the reader.

Reader verification and resynchronization. The tag receives FLAG=1 and 16-bit random number $(R N D 16)$ in step 3, the tag also generates a temporary seed: seed temp. The temporary seed is used to draw a 16-bit random number to verify $R N D 16$. If they are equal, the reader is considered valid. The tag updates its seed to generate the seed'. A 16-bit random number is drawn from seed' to be sent to the reader. Before the tag sends 


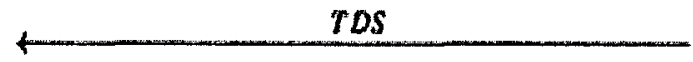
If $\mathrm{TDS}==\mathrm{TDS}_{\text {pre }}$ then
$\operatorname{secd}_{\text {temp }}=P R N G_{80: 111}\left(\operatorname{secd}_{\text {pre }}\right) \ominus \operatorname{secd}_{p r c} \oplus T D$
RND16= PRNG : $15_{15}\left(\operatorname{secd}_{\text {temp }}\right)$

$\operatorname{secd}_{\text {temp }}=$ PRNG $_{80: 111}(\operatorname{secd}) \oplus \operatorname{secd} \oplus \mathrm{TD}$

if $\operatorname{PRNG}_{0.15}\left(\right.$ seed $\left._{\text {temp }}\right)==$ RNDI6 then

$\operatorname{secd}=\mathrm{PRNG}_{16: 47}(\mathrm{seed}) \oplus \operatorname{seed} \oplus \mathrm{TD}$

$\operatorname{secd}^{\prime}=$ PRNG $_{48: 79}($ seed $) \oplus \operatorname{seed} \oplus \mathbf{R D}$

RND16 = PRNG0.15 (seed')

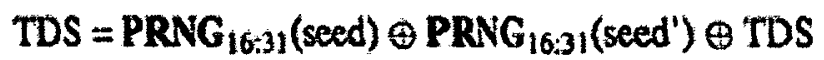

RND16

seed $^{\prime}=\mathbf{P R N G}_{48 ; 79}\left(\right.$ seed $\left._{\text {arr }}\right) \oplus \operatorname{seed}_{\text {aur }} \oplus \mathrm{RD}$

if PRNG $_{0: 15}\left(\right.$ seed' $\left.^{\prime}\right)=$ RNDI6

then Re-synchronize succeeded, Reader sends query to the tag and re-verify it.

Figure 9: The DTN based RFID protocol resynchronization strategy. 
back the random number, it updates its $T D S$.

Tag verification In step 4, the reader generates its seed' to draw a 16-bit random number. If the received number and generated number are equal then the tag is resynchronized with the reader. The resynchronization session is complete. The reader then starts another inventory session for mutual verification.

\subsubsection{Collision resolution}

Since the tag $T D S$ is updated every time after an inventory is performed, it is possible that several tags simultaneously share the same TDS. When a TDS collision occurs, the reader tries to differentiate the tag first and then begins another inventory for mutual verification. This strategy is shown in Figure 10.

Tag and collision identification. If several reader's records have their $T D S_{c u r}$ equal to the received $T D S$ in step 2, a TDS collision occurs. The reader sets the FLAG to 2 to alert the tag. The seed ${ }^{\prime}$ is generated to draw a 16-bit random number (RND16). RND16 is sent to the tag along with FLAG.

Reader verification. In step 3, after the tag receives the message from the reader, it generates the seed ${ }^{\prime}$ to draw the 16-bit random number. If the received 16-bit number and generated number are equal, the reader is considered valid. The tag then generates a temporary seed temp to calculate the seed'. The seed' is used to draw a 16-bit random number to prove the tag identity to the reader.

Tag verification and collision resolution. Similarly, the reader generates the temporary seed temp $_{\text {and then calculates the seed }}$ to draw the 16-bit random number to verify the received one in step 4. If the received 16-bit number and generated number are equal, then 


\section{If TDS collision in the reader then}

$$
\text { seed' }=\mathbf{P R N G}_{48: 79}\left(\text { seed }_{\text {eur }}\right) \oplus \operatorname{seed}_{\text {our }} \oplus \mathbf{R D}
$$

RND16 $=$ PRNG $_{32 ; 47}($ seed')

$$
\begin{aligned}
& \operatorname{sed}^{\prime}=\mathbf{P R N G}_{48,79}(\text { seed }) \oplus \operatorname{secd} \oplus \mathbf{R D} \\
& \text { if } \operatorname{PRNG}_{32: 47}\left(\operatorname{secd}^{\prime}\right)=\text { RNDL6 then } \\
& \text { seed }_{\text {temp }}=\mathbf{P R N G}_{16 \mathrm{~A} / 7}(\mathrm{secd}) \oplus \operatorname{seed} \Theta \mathrm{TD} \\
& \text { seed }^{\prime}=\text { PRNG }_{48: 79}\left(\text { seed temp }_{\text {to }} \text { seed }_{\text {temp }} \oplus \mathbf{R D}\right. \\
& \text { RNDI6 = } \text { PRNG }_{4863} \text { (seed) }
\end{aligned}
$$

seed $_{\text {temp }}=$ PRNG $_{16: 47}($ seedas $) \oplus \operatorname{secd}_{\mathrm{axi}} \oplus \mathrm{TD}$

seed" $=$ PRNG $_{48779}\left(\right.$ seed $\left._{\text {temp }}\right) \oplus \operatorname{secd}_{\text {temp }} \oplus$ RD

If PRNG $_{48: 63}\left(\right.$ seed $\left.^{\prime}\right)=$ RND16 then

Find the lag's information, stari another inventory to verify this tag else

Choose another collided tag to calculate the PRNG 4263 (seed') again

Figure 10: The DTN based RFID protocol collision resolution strategy. 
the chosen tag is the correct one. If the generated 16-bit random number does not equal the received one, the reader chooses another collided tag to re-calculate the 16-bit number.

This strategy requires the tag to update the $\operatorname{seed}_{c u r}$ to generate a new seed' temporarily. The seed' is used to draw the 16-bit number used by the reader to differentiate the tag. Since the $T D$ is uniquely assigned to each tag, by updating the $\operatorname{seed}_{c u r}$ to $\operatorname{seed}_{t e m p}$ with the $T D$, the updated seed temp are different between collided tags. Thus, the seed that is generated from the seed $d_{\text {lemp }}$ and the 16-bit random number drawn from the seed' can differentiate collided tags.

During a collision situation or a de-synchronization, readers and tags need to recover to normal state before beginning a new mutual verification session. An adversary cannot replay the collision message or the de-synchronization message for mutual verification. Once a tag is synchronized with a reader, the de-synchronization alert message becomes invalid. If an adversary re-sends the collision alert message to the tag, the tag replies with the same message it sent to the reader before. Furthermore, adversaries cannot alert a new de-synchronization or collision without obtaining a valid reader's $P R N G$ seed, tags need to verify the reader before replying or changing their local state. 


\section{Chapter 4}

\section{Analysis and delay tolerant network}

In this chapter, we first discuss available $P R N G$ functions and data deployment strategies for our proposed protocol. Security is also analyzed against relevant attacks in the second part of this chapter. In the final part of this chapter, Delay Tolerant Network is studied as a message transmission media to synchronize RFID readers.

\section{1 $P R N G$ implementation}

The $P R N G$ function takes an initial set of values to generate a sequence of numbers having approximately random properties. The set of values is called a $P R N G$ seed. The sequence of numbers is reproducible, when the same $P R N G$ seed is provided. In our proposed protocol, readers and tags keep the same $P R N G$ seeds locally to generate identical number sequences for mutual verification. EPCglobal standards provide specific requirements for the $P R N G$ function without a specific algorithm for implementation. Several efficient $P R N G$ function algorithms are proposed and discussed for lightweight RFID tags. The self-shrink $P R N G$ [21] was developed from the shrinking PRNG [9]. It has been implemented for RFID tags. It is estimated to use 1435 logic gates and 64bytes of memory [20]. More recently, the EPCglobal standard compatible PRNG function LAMED-EPC [23] has been

proposed. It requires 1566 logic gates and 64bytes of memory. Both of these two PRNG 
Table 7: Inventory commands (they are all mandatory).

\begin{tabular}{l|l}
\hline Command & Length (bits) \\
\hline \hline Query & 22 \\
\hline QueryAd just & 9 \\
\hline QueryRep & 4 \\
\hline Query_Reply(RND) & 16 \\
\hline ACK & 18 \\
\hline ACK_Reply & 21 to 528 \\
\hline NAK & 8 \\
\hline \hline
\end{tabular}

function can be utilized within our proposed protocol.

\subsection{Compatibility analysis}

\subsubsection{Protocol compatibility}

The EPCglobal standard specifies a communication between a reader and a tag into three processes:

- Select. This process is for a reader to select a tag population defined by user specified criteria. This command is mandatory before an inventory and access round.

- Inventory. This process is for a reader to identify a tag. Inventory commands include Query, QueryAdjust, QueryRep, Query_Reply(RND), ACK, ACK_Reply and NAK. Our proposed protocol inherits those commands to achieve mutual verification without changing their original functions. Lengths of inventory commands are given in Table 7. All current commands are mandatory.

- Access Commands 


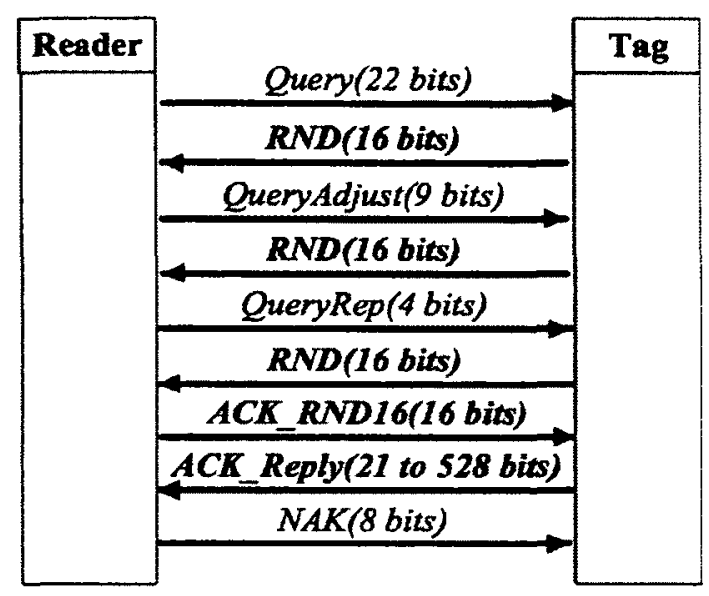

Figure 11: The EPCglobal standard inventory protocol.

Access Commands execute actual interactions between a reader and a tag. A tag can be read, rewritten, updated or killed. Access commands can only be used after an inventory session.

A full detailed EPCglobal inventory protocol is shown in Figure 11. Our proposed protocol is shown in Figure 12.

In the EPCglobal inventory protocol, the reader sends commands Query, QueryAd just, QueryRep to conduct a random-slotted collision algorithm. First, the reader sends the Query that contains a parameter $Q$ to the tag. The tag generates a random $Q$-bit number locally as a counter and a 16-bit random number $R N D$. It replies the $R N D$ as an acknowledgement to the reader. Second, one or several QueryAd justs can be sent from the reader to adjust the tag's counter value. Every time, the tag replies the same $R N D$ to the reader as an acknowledgement. Third, the tag begins to decrease its counter when it receives the QueryRep. The tag sends the same RND back to the reader when the counter reaches zero. The reader then sends back the same $R N D$ to the tag as $A C K$. If the tag does not receive a valid $A C K$ due to a collision, then it returns to the initial state and repeats the process. Otherwise, the tag sends back an $A C K \_$Reply including its $E P C$ identification. At the end, 


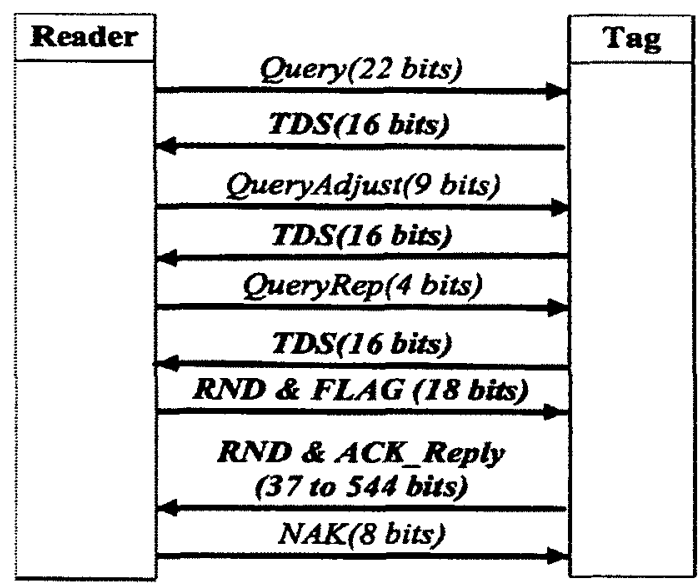

Figure 12: The DTN based RFID protocol.

the reader replies a $N A K$ to complete the inventory round.

As defined in Table 7, support of all EPCglobal inventory commands are mandatory. In our proposed protocol, these commands are adopted with minor modifications. TDS is used to replace the 16-bit random number $(R N D)$ sent from the tag to the reader to echo Queries. The $A C K$ sent from the reader is replaced by a 18-bit number including a calculated 16-bit random number and a 2-bit FLAG. We also extend the ACK Reply message to include a 16bit random number for tag identity verification. The three $Q u e r i e s$ and the NAK commands are kept the same for the random-slotted collision algorithm.

\subsubsection{Data compatibility}

The EPCglobal standard specifies four data banks for RFID tags [13].

Reserved memory (bank00). This bank of data is reserved for the kill password and access password. The kill password is used for permanently terminating the use of one tag. The access password is used by the reader to perform privileged operations. Those two passwords are used by access commands. 
EPC memory (bank 01). This data bank stores EPCglobal protocol-control information.

TID memory (bank 10). This data bank keeps the TID (Tag Identification). The EPCglobal standard outlines two schemes to identify a tag:

- 32 bits Short Tag Identification. It includes a 12-bit tag MDID (Tag Mask Designer Identifier), a 12-bit tag model number and a 8-bit class identifier.

- 128 bits Extended Tag Identification. The first 32 bits are the same as the short tag identification. The remaining 96 bits (Extended TID) are kept for end users and RFID applications. The Extended TID memory section can be partitioned into six segments. Each segment has16 bits.

User memory (bank 11). This data bank is optional. It provides user defined data storage for EPCglobal applications.

\subsubsection{Data deployment}

Based on the EPCglobal standard, the possible data memory deployment for our proposed protocol is as follow.

$R D$ (32 bits), TDS (16 bits) and seed (32 bits).

$R D$ is an identity of readers. When it is configured into a tag, it is stored permanently. $T D S$ is the pseudonym number. seed is a $P R N G$ seed. Their initial value is configured into a tag and is updated after every inventory. These three 16-bit numbers can be stored in three segments of the extended TID in the TID memory (bank 10). 
$T D$ (32 bits).

The $T D$ is an unique identity for a tag in the DTN based RFID protocol. According to the EPCglobal standard, the TID is different for tags with different custom commands and/or optional features. If each tag has unique features and/or RFID commands, then the DTN based RFID protocol can use the TID as TD. If tags have common commands or features (e.g., cell phones have same features or functions but they need to be treated individually for different customers), then the EPCglobal tag URI can be used. The EPC $U R I$ scheme is used within an information system to represent each physical object. In our proposed protocol, the URI scheme can be used to differentiate all tags. The URI format is defined as urn:epc:id:scheme:component 1.component 2 . The urn:epc:id:scheme is the URI body. It indicates the URI scheme. The component 1 and component 2 are the remainders of the scheme. The EPC URI currently supports ten schemes. The sgtin scheme is typically used for trading item. Each object has an unique identity. The general syntax of sgtin scheme is urn:epc:id:sgtin:CompanyPrefix.ItemReference.SerialNumber. The CompanyPrefix represents a managing entity. The ItemReference is assigned by the managing entity to a particular object class. The particular object class gives each individual object an unique SerialNumber within its scope. Following this scheme, in an user specified criteria, tags can be divided into groups by assigning different 32-bit CompanyPrefix and ItemReference. Each tag is assigned an unique 32-bit SerialNumber within one group. The SerialNumber can be used as tags TD. The ItemReference and CompanyPrefix can be used as $R D$. Thus, tags in the same group share a $R D$ but they have different $T D$ s. Readers use the $R D$ to access a group of tags and use a $T D$ to verify the identity of an individual tag. 


\subsection{Security analysis}

The DTN based RFID protocol meets the following security requirements: mutual verification, data confidentiality, tag anonymity and untraceability, forward security and backward security.

\subsubsection{Mutual verification}

Readers and tags need to verify each other before starting a communication. Mutual verification is achieved by using the same $P R N G$ state: Readers and tags keep a same PRNG function and $P R N G$ seeds to generate identical random number sequences for mutual verification. After every successful inventory, the PRNG seeds are updated for the next inventory. Thus, as long as the $P R N G$ states remain synchronized, tags and readers can verify each other.

\subsubsection{Data confidentiality}

All transmission messages between tags and readers are random numbers. With a synchronized $P R N G$ states, valid readers and tags can recognize those messages and verify each other. Since the PRNG seed is updated after every communication, the random number sequence is different in each inventory.

\subsubsection{Tag anonymity and untraceability}

In our proposed protocol, a tag sends a pseudonym number (TDS) instead of the real identity $T D$ for identification. The $T D S$ is updated every time after a successful inventory. Readers need to use a different $T D S$ to identify the same tag in the next inventory. A tag's identification is hidden to the environment and its anonymity is guaranteed. 


\subsubsection{Forward security}

Forward security is defined in [5] as: previous messages of a tag need to look random even if the tag is corrupted. In the DTN based RFID protocol, if a tag is compromised, its local parameters (TD, RD, TDS, seed) are exposed to the adversary. The $P R N G$ seed is updated as followed:

$$
\operatorname{seed}_{c u r}=T D \oplus \operatorname{seed}_{c u r} \oplus P R N G_{16: 47}\left(\text { seed }_{\text {cur }}\right)
$$

According to this equation, seed $_{c u r}$ is updated using the previous seed $d_{c u r}$. The previous seed is erased. Adversaries require previous seeds to regenerate previous messages. Since tags do not store previous seeds, adversaries cannot generate previous messages and forward security is achieved.

\subsubsection{Backward security}

Backward Security is defined as: following the update of a tag by a reader, future communication messages look random to adversaries, even when they have access to a tag's current state [5]. In the DTN based RFID protocol, once a tag is updated, a new PRNG seed and a new $T D S$ are generated for the upcoming verification. The previous PRNG state and $T D S$ are invalid and adversaries cannot use them to decipher future messages.

\subsection{Delay tolerant network}

\subsubsection{Overview}

Most proposed RFID protocols use backend server connections to synchronize RFID readers. In some scenarios, it is not practical to use stationary readers with permanent backend server connections. For example, in a warehouse with many products on shelves, it is more 
convenient for an employee to use a portable reader to check and update products information. In order to avoid permanent backend server connections, we use the Delay Tolerant Network (DTN) to connect readers.

Following each successful inventory, a tag updates its $P R N G$ seeds and TDS, while the reader generates a new message and synchronizes with other readers. The new generated message includes the tag's new TDS, previous TDS and two PRNG seeds. Whenever two readers are within each other's communication range, they exchange new messages. Readers update local tag records according to received messages and keep a list of the latest tags' information to share with other readers. Each message is assigned a message Time To Live (TTL). A message is dropped when its TTL expires.

\subsubsection{Delay-tolerant networking}

The DTN [16] addresses the message transmission between different nodes in a network which lacks continuous connectivities. Routing protocols in a DTN need to store and forward messages in order to increase the dissemination of information. No routes are pre-established. A DTN has several regions. Each region has different nodes that share a unique region $I D$. A node name consists of two parts: entity $I D$ and region $I D$. Nodes only communicate with those that have the same region $I D$. There are region gateways that can bridge different regions. Within one region, nodes use entity IDs to differentiate each other.

In our proposed protocol, readers are DTN nodes that are responsible to receive, store and transmit new messages. Messages that are generated by the readers are not given specific destination. They need to be sent to all nodes with the same delivery priority. Readers only keep the most recent messages and relay them to other nodes.

Tags can be divided into several regions by assigning different region $I D$ s. A region $I D$ is used as $R D$ in our proposed protocol. A valid reader can check a group of tags and a tag can verify a reader with the region $I D$. Within one region, each reader also keeps an unique 
entity $I D$ to differentiate each other and transmit messages. When a new reader needs to join one existed region, it is configured with the region $I D$ and an unique entity $I D$.

\subsubsection{DTN message routing}

All the readers in the same region need to update their local records when there is a new message generated. All readers are destinations for the message. The message routing protocol does not need to provide a one-to-one routing strategy. We assume that positions of portable readers change all the time. Therefore no fixed path can be determined. No movement patterns can be predicted. The epidemic routing protocol [31] is considered as a possible routing strategy for the DTN based RFID protocol. Packets are received at intermediate nodes and forwarded to all the node's neighbours except the one who sent the packet.

\subsubsection{DTN and the DTN based RFID protocol}

Our proposed protocol is conducted in the EPCglobal inventory session between the reader and the tag. A DTN is only used between RFID readers, RFID tags are not participants. Every time after a reader checked a tag, new $T D S_{c u r}, T D S_{p r e}$, seed $_{c u r}$ and seed pre $_{\text {are gen- }}$ erated and need to be synchronized to other readers to make the tag readable again. The DTN is a media between readers to exchange new messages and all DTN communications are assumed to be secure. Each RFID reader is a DTN node and can be used at random locations. After a RFID reader receives a message, it updates its local records to synchronize with the correlated RFID tag. 


\section{Chapter 5}

\section{Simulation}

In this chapter, the simulator - Opportunistic Network Environment (ONE) [19] is used to emulate the RFID readers communications.

\subsection{Introduction}

In simulator $O N E$, three parts of a DTN are simulated: node compatibility, node mobility and message routing. The node compatibility enables nodes to act as a store and carry router using different routing algorithms. Certain parameters are provided to simulate: network interface, persistence storage, message routing and energy consumption.

Routing strategies, for messages in a DTN, include epidemic routing [31] and spray and wait routing [30].

$O N E$ provides map-based mobility and working day movement model for node mobility simulation. For map-based mobility, nodes are only allowed to move on pre-defined routes with different movements strategies including random movement, shortest path movement and routed movement. The random movement mode allows nodes to walk randomly on the map. The shortest path movement randomly chose a destination from the map. Between the previous destination to the next destination, nodes take the shortest path 


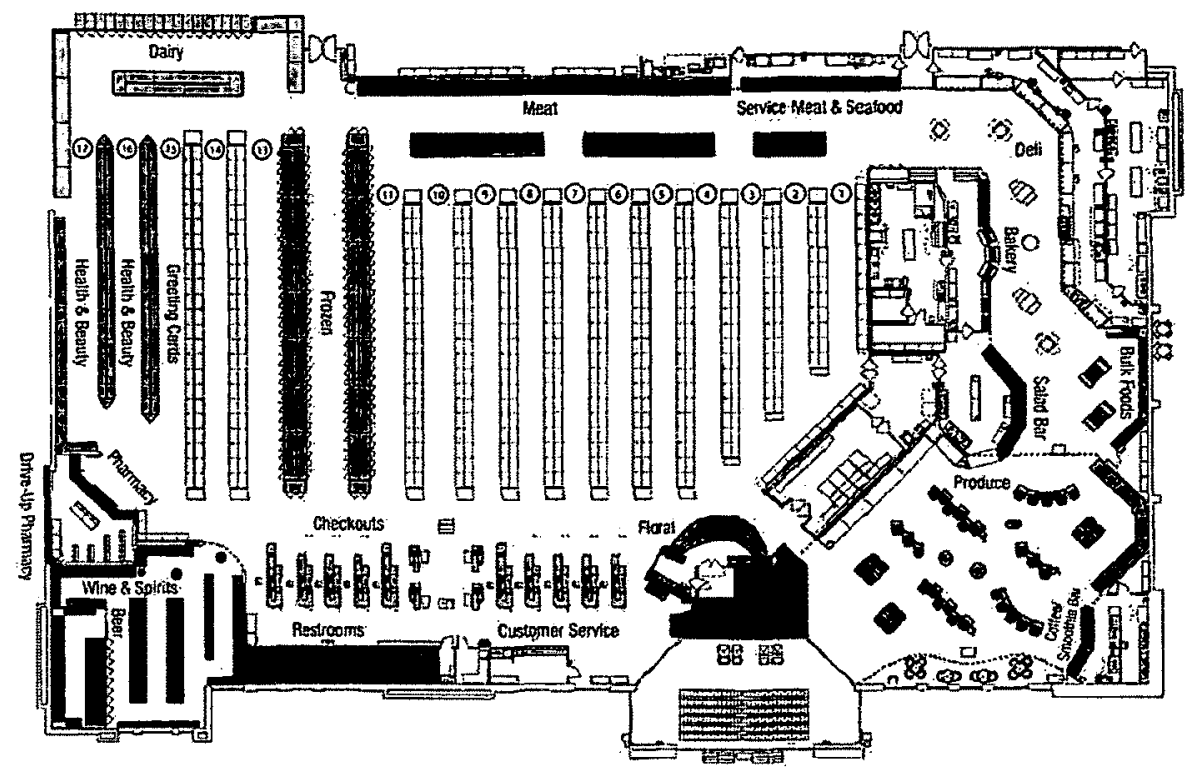

Figure 13: The map of a supermarket.

and stay at one destination for a random time interval. The routed movement uses predetermined routes for nodes. The working day movement model consists of several sub models that define daily routings at different time of a day for objects within a city.

\subsection{Scenario}

Readers communicate with each other within a DTN. Whenever a tag is read by a reader, a new message is generated. It is sent to other readers to make the tag readable again. We describe a supermarket scenario. There is a mall with goods and products that are equipped with RFID tags. Readers are used by workers to manage different product information. An example supermarket map is provided for this scenario in Figure 13.

Based on the map, the available routes are drawn as Figure 14 for reader holders to walk. The size of this supermarket is approximately 3,700 square meters. Each node has a 10 meter communication range. They can communicate with each other using a bluetooth 


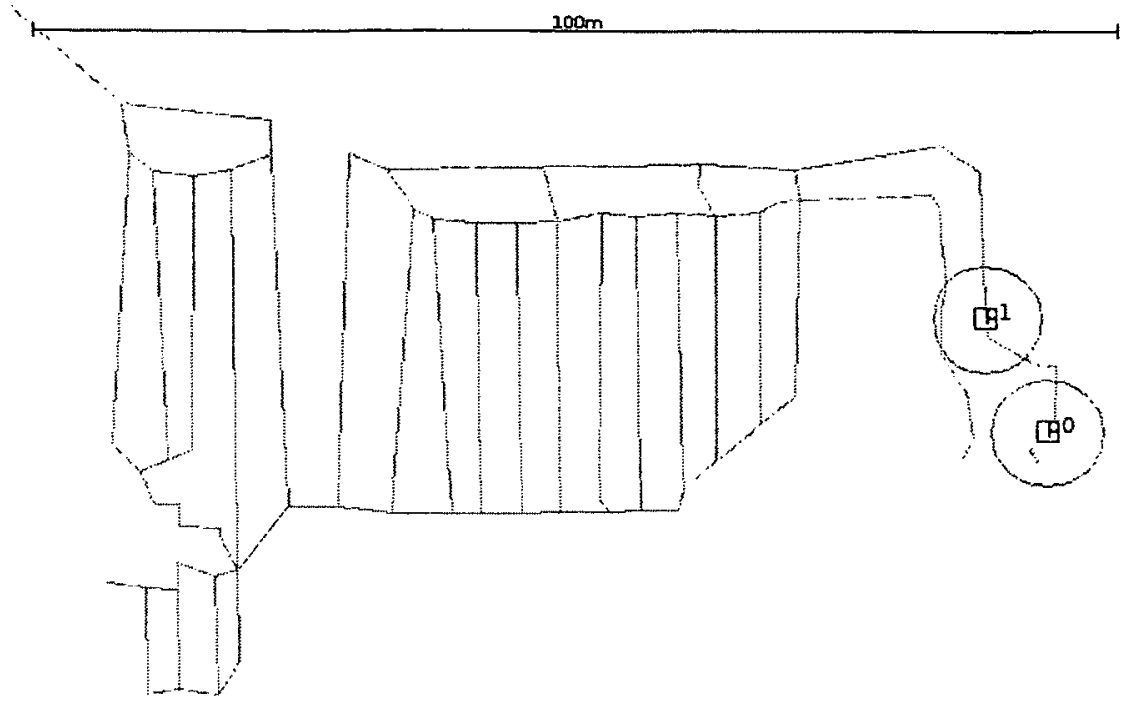

Figure 14: The route map.

network interface.

All the reader holders walk randomly on the route defined in Figure 14. Once they reach a destination by taking the shortest path, they remain there for a random interval of time. Then, the next destination is randomly determined.

In this scenario, the main purpose of RFID reader holders is to check or update certain product information with a reader. Therefore, a new message is generated with the updated information regarding the tag every time a reader holder reaches a destination. Those new messages are broadcasted between different readers whenever they are within each others range. Each reader only updates new messages according to their local records and discards those messages whose TTL is expired.

All the readers in the simulation use a transmission speed of $250 \mathrm{KBps}$. A reader's buffer size is $5 \mathrm{M}$ bytes, while a single updated tag message is 160 bits. Since all the messages are assigned a same TTL. They are discarded when their TTL is expired. It is assumed that readers have enough space to store all incoming messages until they become expired. 
Each reader holder walks at a randomly chosen speed between 0.5 to 1.5 meters per second. Once a reader holder arrives at its destination, it stays there for a random time, between 0 to 200 seconds.

\subsection{Performance analysis}

Each message contains update information for a reader's local tag record. Reader needs to receive the message and update the tag to make it readable again. We say a message is synchronized, if all readers receive this message and update its local record within one DTN region. Two parameters are defined to evaluate the result of this simulation: Message Coverage Rate and Message Update Success Rate. The Message Coverage Rate indicates the average percentage of synchronized messages to the overall number of messages among all nodes, at a certain time point. The Message Update Success Rate shows the percentage of messages that have been sent to all nodes before they are dropped. Readers require a high Message Coverage Rate to stay synchronized. They also require a $100 \%$ Message Update Success Rate to ensure that all messages can be sent to all readers before they are dropped. In order to show the influence of the message traffic, the same simulation is conducted under two different situations: $100 \%$ message generation and $50 \%$ message generation. $100 \%$ message generation enables readers to generate a message every time they stop at a destination while the $50 \%$ message generation only gives $50 \%$ possibility to a reader to generate messages when it arrives at a destination.

Figure 15 and 16 show the total number of messages that are generated before they reach the TTL and begin to be dropped. The number of messages from scenarios with three, six and nine nodes are demonstrated together at different time from 300 s to $2400 \mathrm{~s}$. The TTL is longer than 2400 , no messages are discarded in these two figures. The number of message is growing as time goes by. Those two figures also show the difference of the number of messages from the $100 \%$ message generation In Figure 15 and the $50 \%$ message 


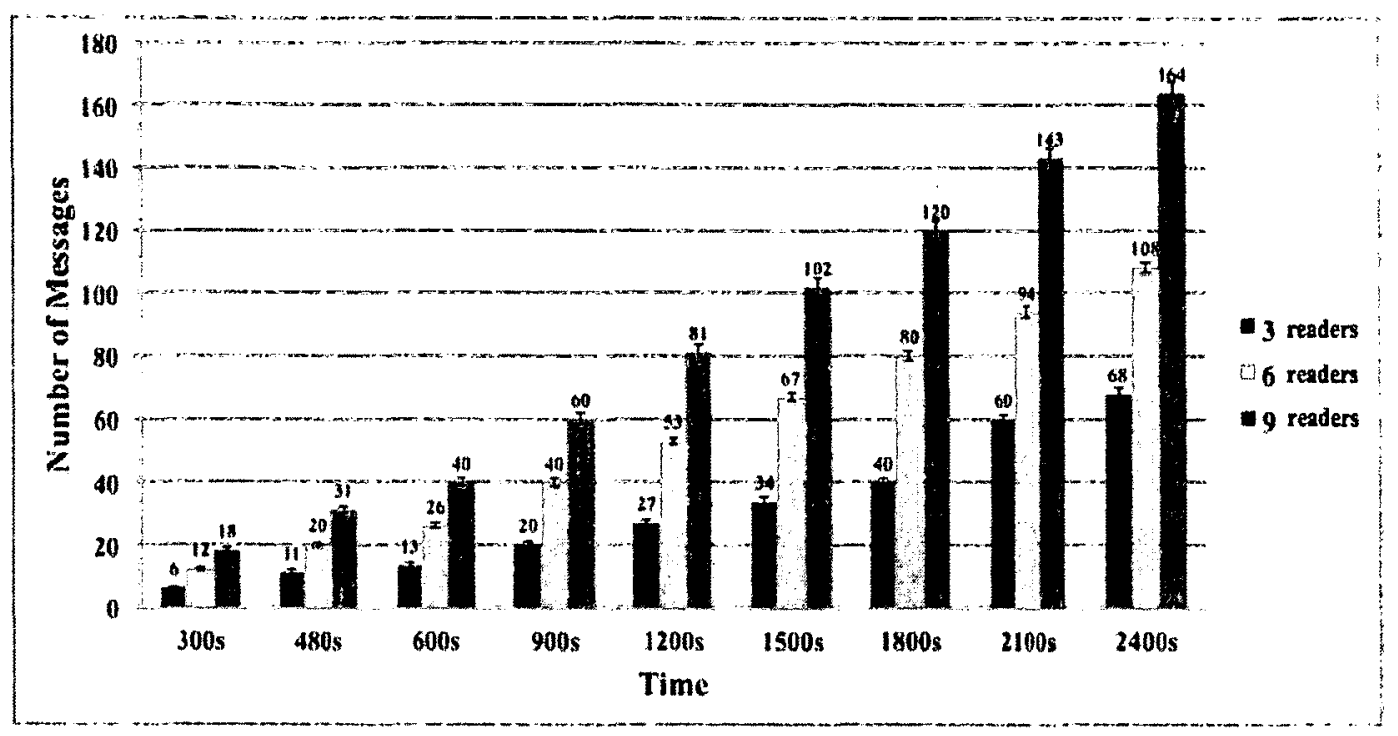

Figure 15: $100 \%$ message generation - The total number of generated messages with the $90 \%$ confidence interval.

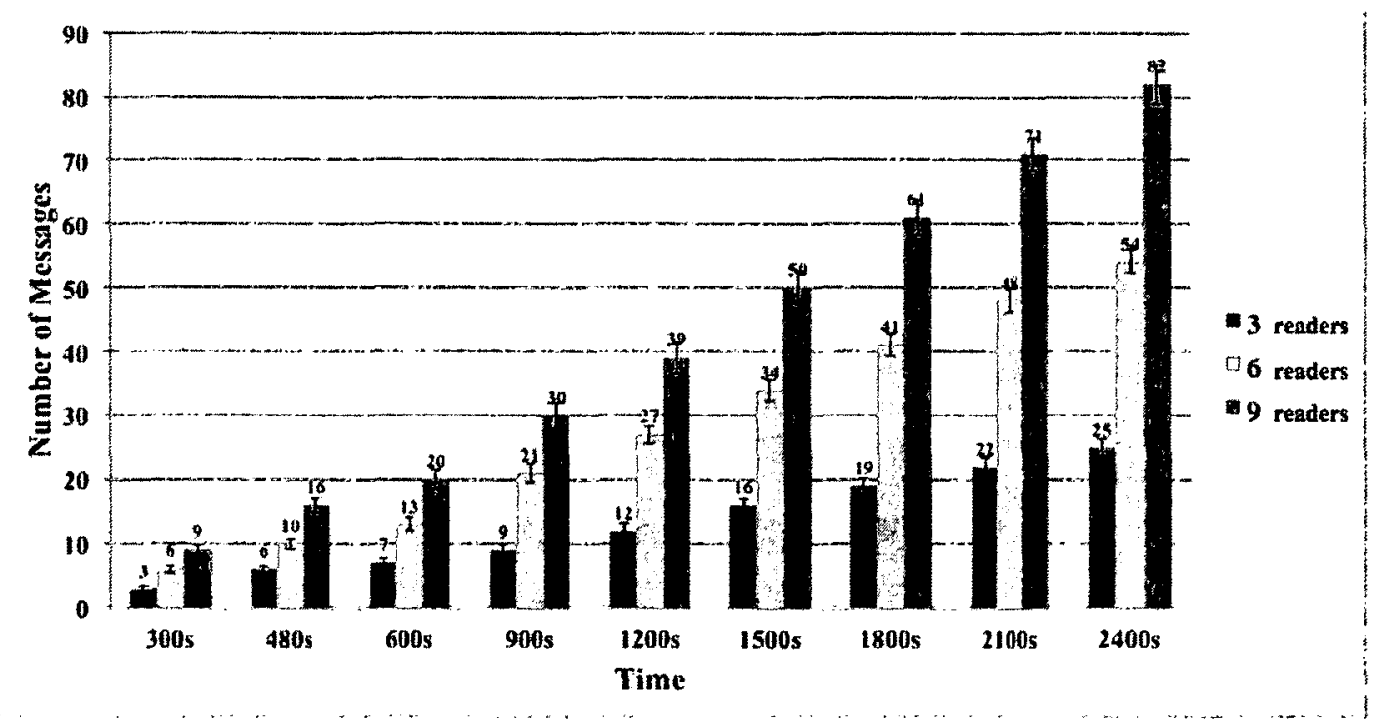

Figure 16: $50 \%$ message generation - The total number of generated messages with the $90 \%$ confidence interval. 
generation, the formal one generates approximately twice number of messages of the later one.
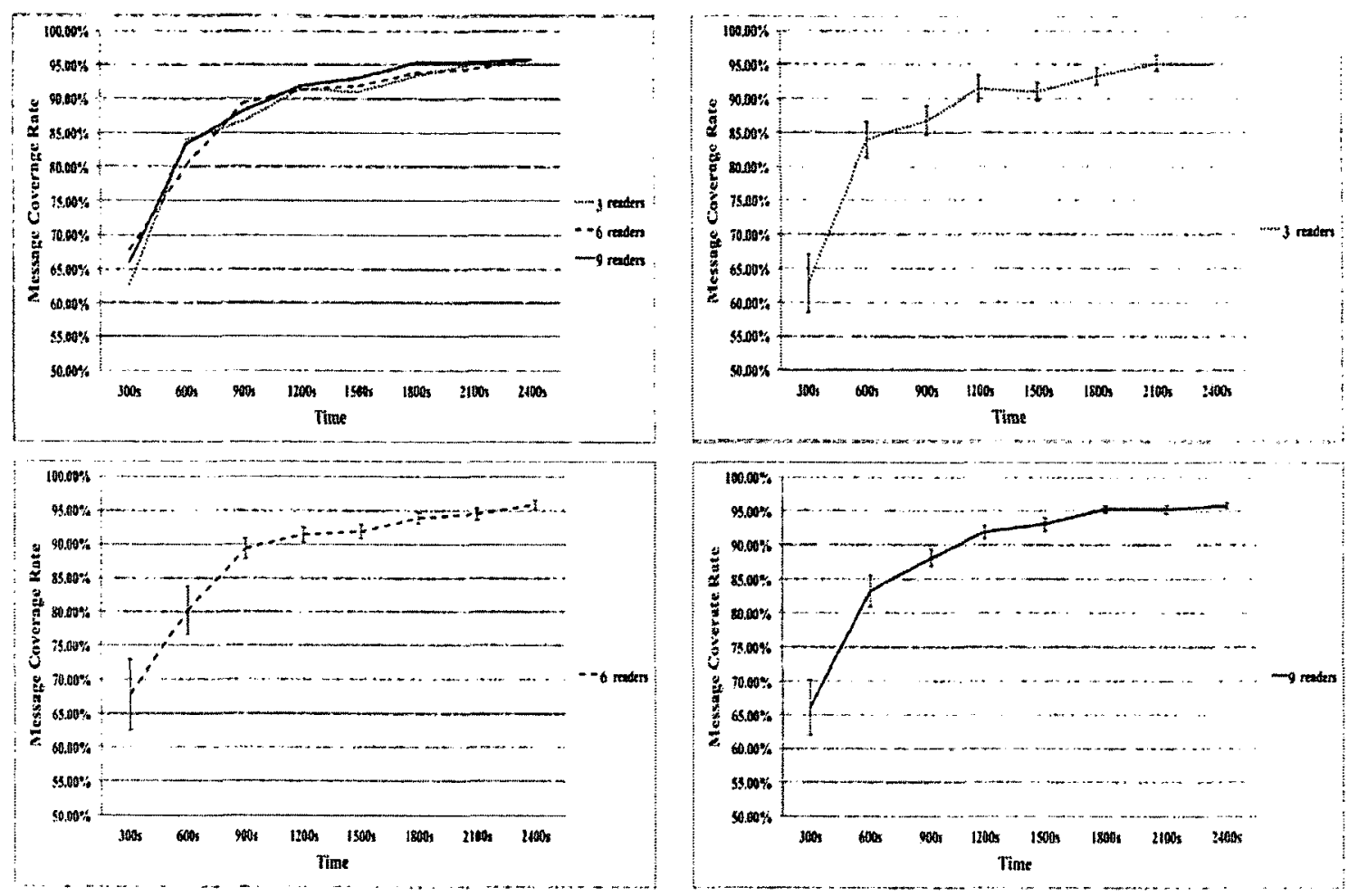

Figure 17: 100\% message generation - Message Coverage Rate with the $90 \%$ confidence interval.

As it is shown in Figures 17 and 18, 90\% of messages are synchronized among all readers after 20 to 30 minutes. Moreover, different numbers of reader holders in the environment have similar performance to each other. The high percentage of synchronized messages remains stable between $90 \%$ to $95 \%$ after 45 minutes.

Figure 19 and 20 show the influence on the Message Update Success Rate between different message TTLs, reader populations and message traffic. It turns out that when the TTL is set to 15 minutes, some reader messages fail to update reader's local records before those messages are dropped. This simulation shows that the higher the Message Coverage Rate is, the higher the Message Update Success Rate will be. 

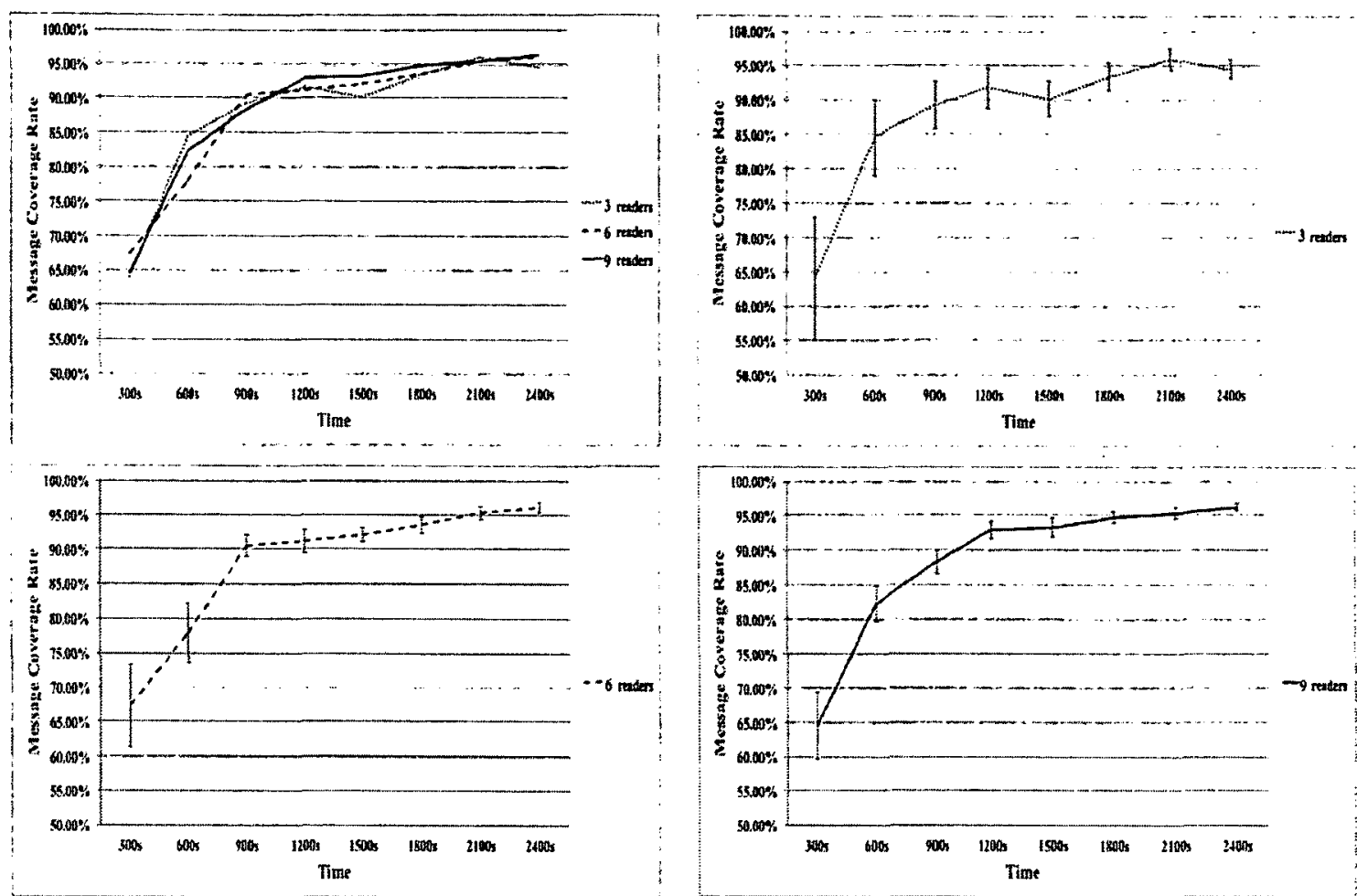

Figure 18: $50 \%$ message generation - Message Coverage Rate with the $90 \%$ confidence interval.

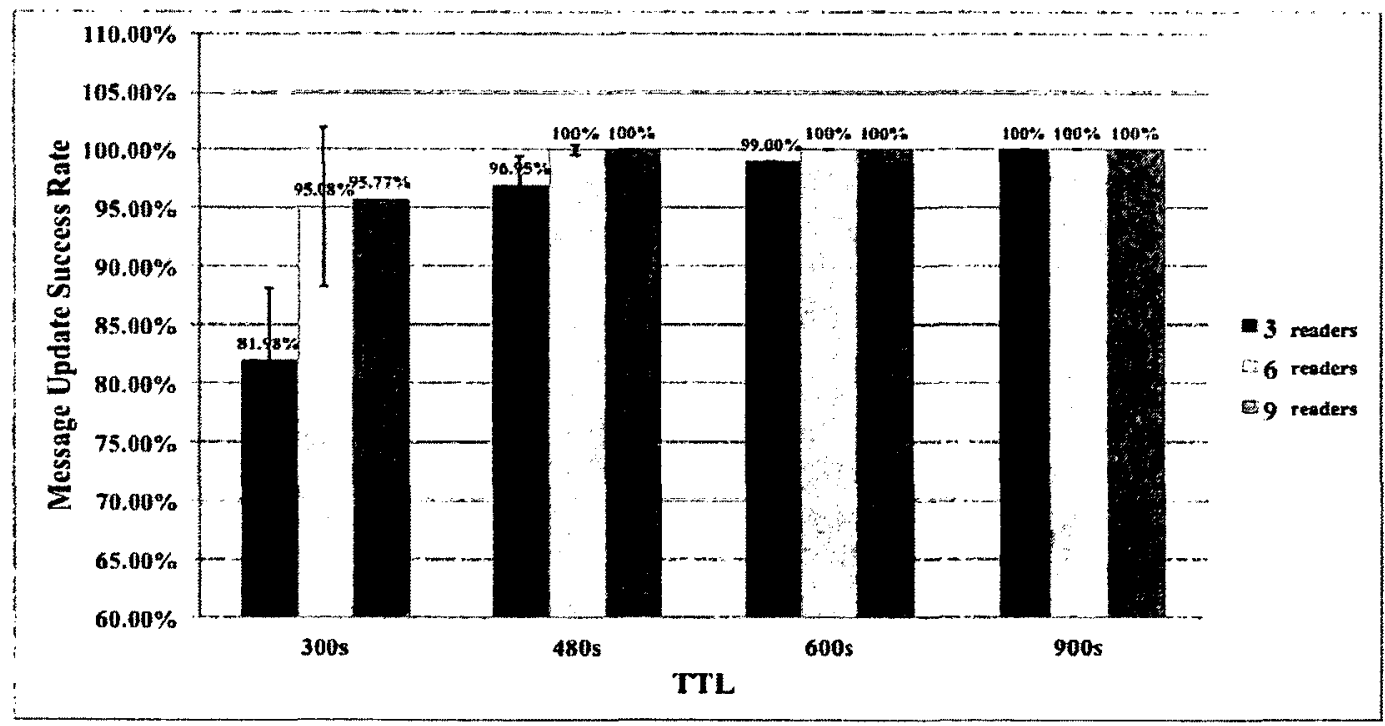

Figure 19: $100 \%$ message generation - Message Update Success Rate with the $90 \%$ confidence interval. 


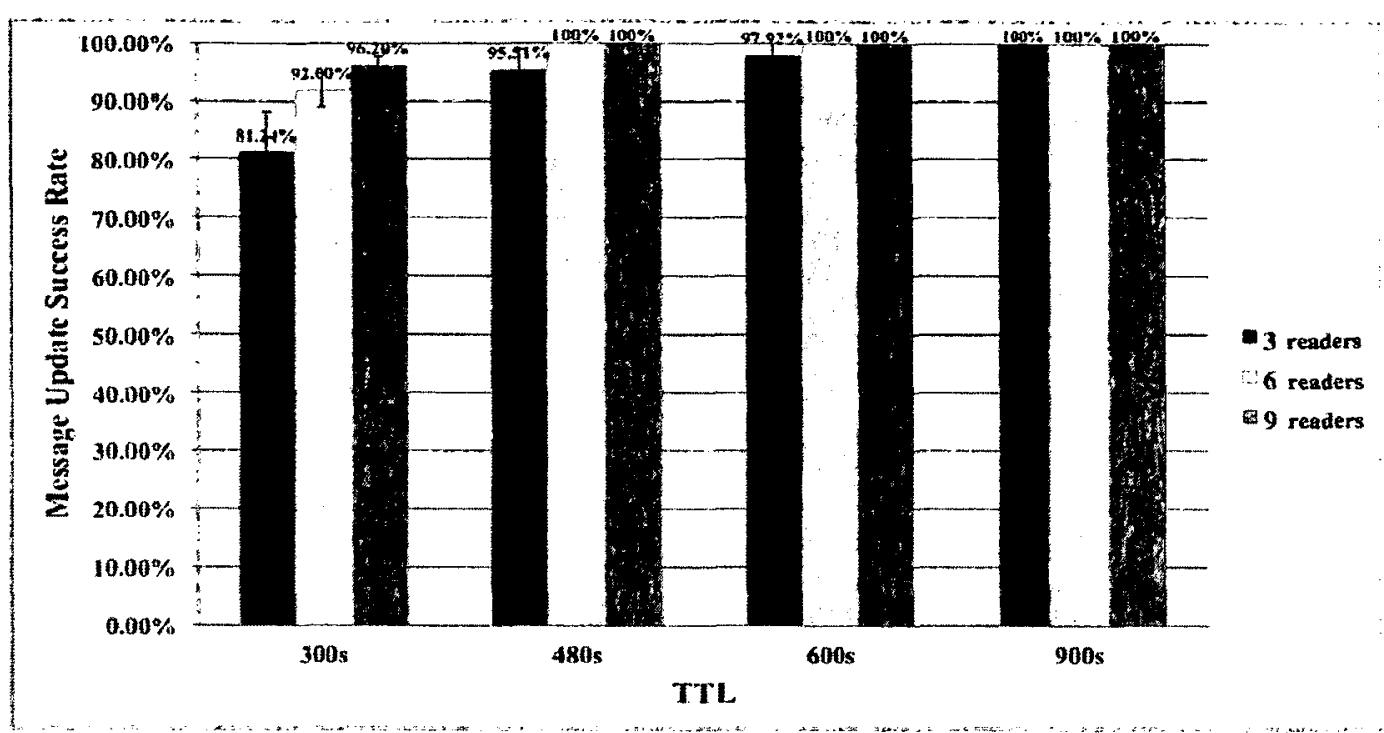

Figure 20: 50\% message generation - Message Update Success Rate with the $90 \%$ confidence interval.

Based on our simulation results differences in the number of messages and reader holders do not significantly affect the Message Update Success Rate and Message Coverage Rate. The Message Update Success Rate mainly depends on the TTL. The longer the TTL, the higher the possibility that a message can be sent to all readers. Figures 19 and 20 indicate that it is safe to set the TTL to 30 minutes to ensure $100 \%$ success rate.

The next scenario uses a food warehouse to show the influence of different map sizes. Figure 21 shows a layout of a warehouse. Figure 22 shows the route that reader holders walk. The warehouse size is three times of the size of the supermarket. In Figure 22, a circle represents the transmission range of a node. Each square represents a reader.

Simulation parameters of this warehouse scenario are the same as the supermarket, with the only difference being the size and layout of the map. Figure 23 shows the total number of messages generated in three, six and nine readers scenarios.

Since the warehouse size is three times the one of the supermarket, readers have less 

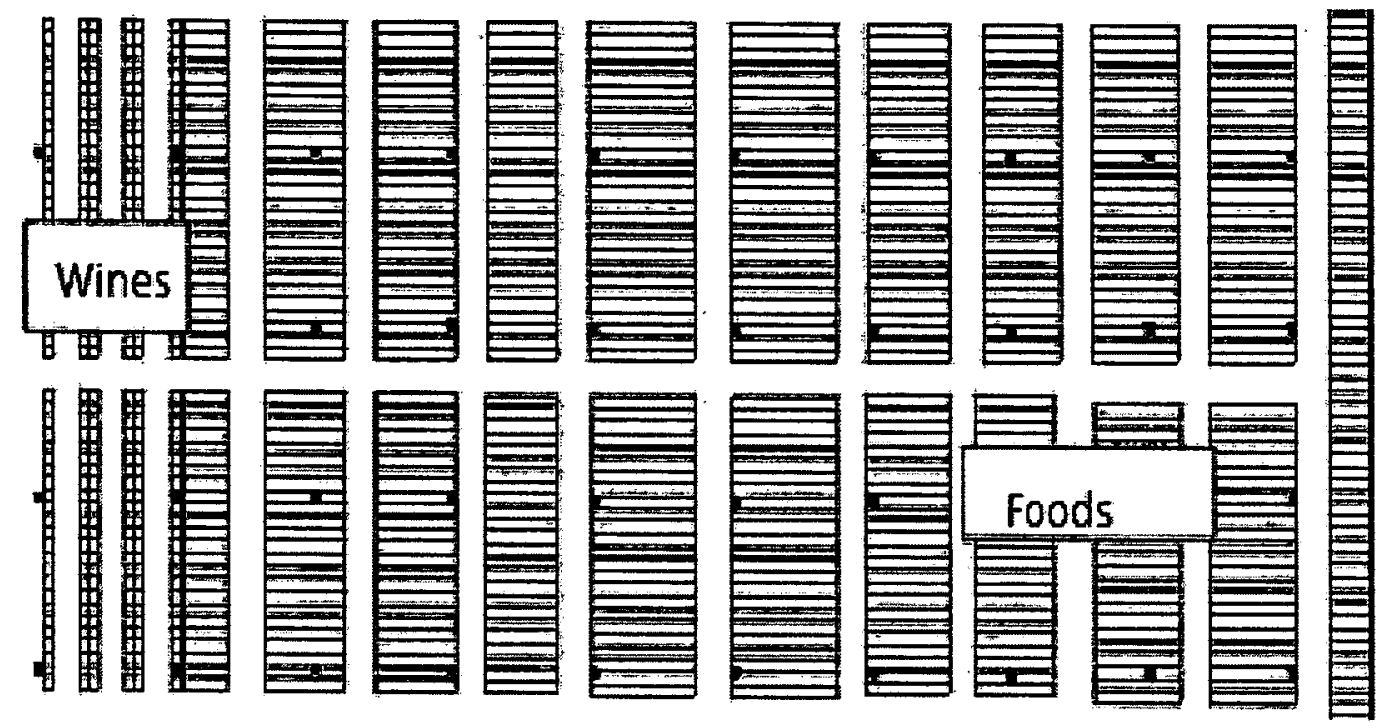

Figure 21: The map of a warehouse.

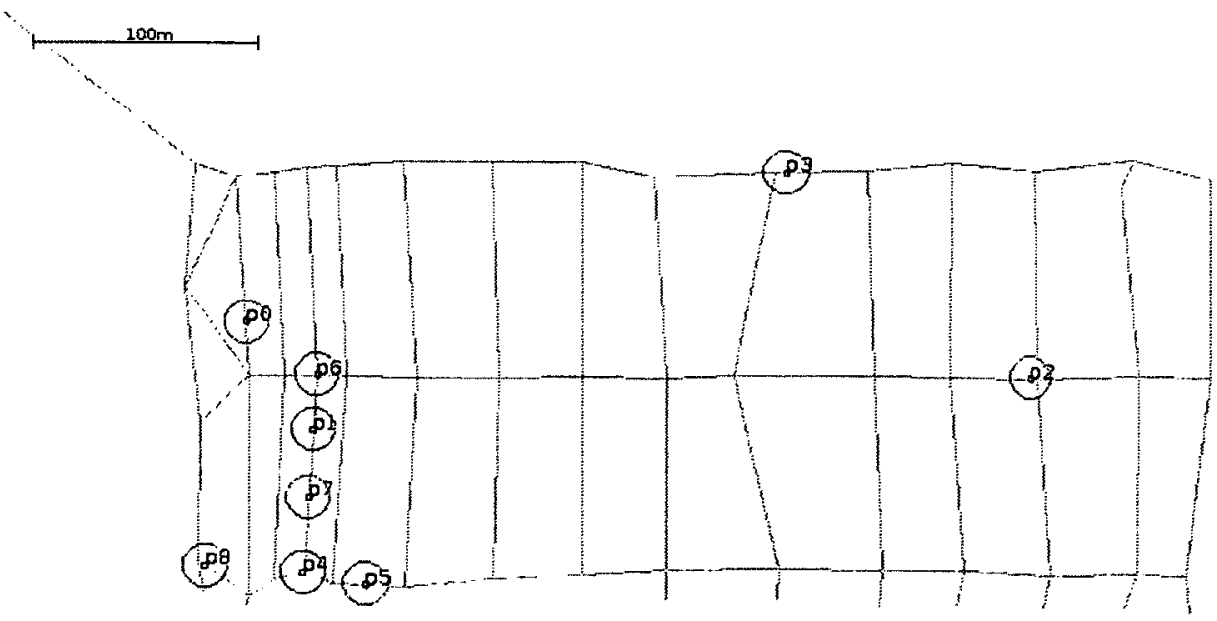

Figure 22: The route map. 


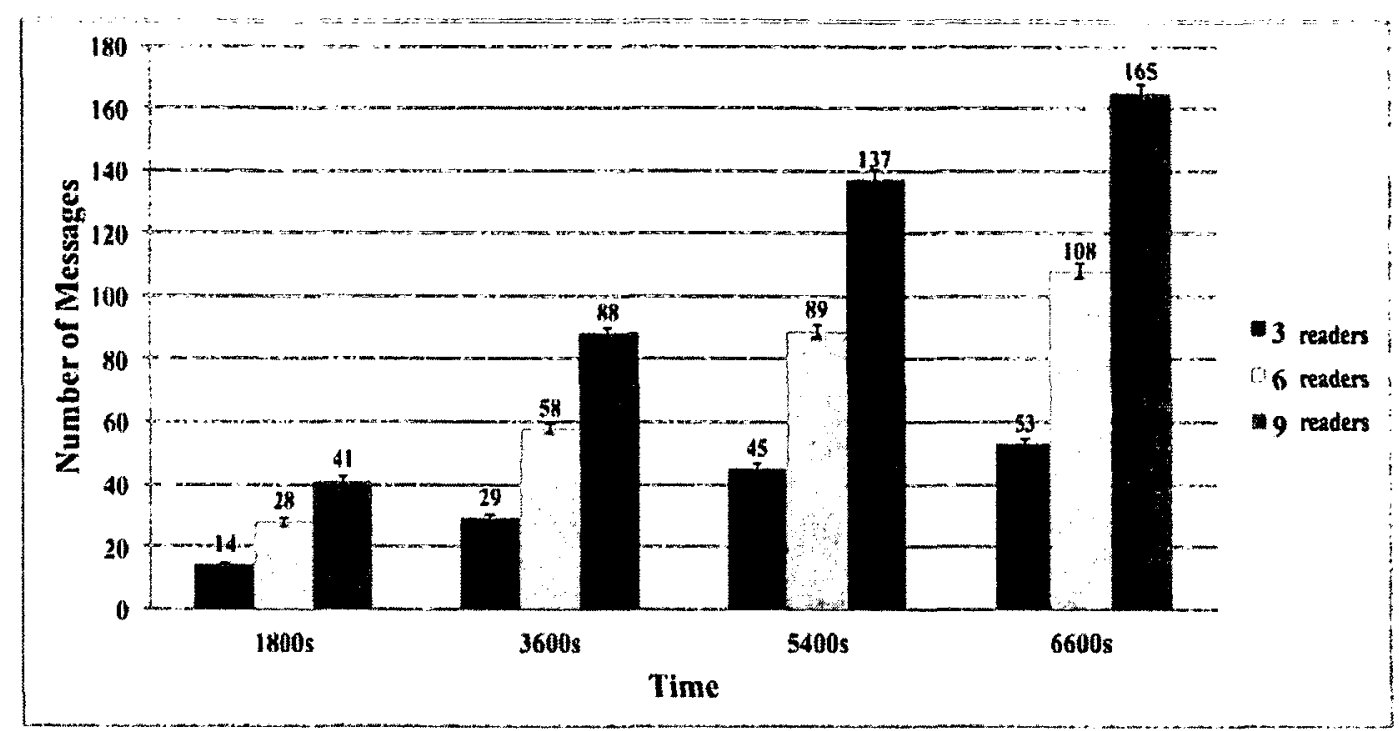

Figure 23: The total number of generated messages with the $90 \%$ confidence interval.

possibility to communicate with each other for a same time period and same node population. A longer time period is used in this simulation to show the result of Message Coverage Rate in Figure 24 and the result of Message Update Success Rate in Figure 25.

Similar to the supermarket simulation, the trend of the Message Update Success Rate and Message Coverage Rate starts with low rates and moves to higher rates. The larger map size slows the overall trend, therefore readers need a longer TTL to achieve the $100 \%$ Message Update Success Rate. If more readers are placed in the simulation, more possibilities are provided for communication. Therefore shorter TTLs are needed to achieve the 100\% Message Update Success Rate. Figure 24 shows that the Message Coverage Rate in a nine node scenario is higher than in both six and three node scenarios; while the six node scenario Message Coverage Rate is higher than the three node scenario. Also from Figure 25 , nine and six node scenarios can reach $100 \%$ Message Update Success Rate with a TTL of 3600 seconds while the three node scenario needs 5400 seconds. 

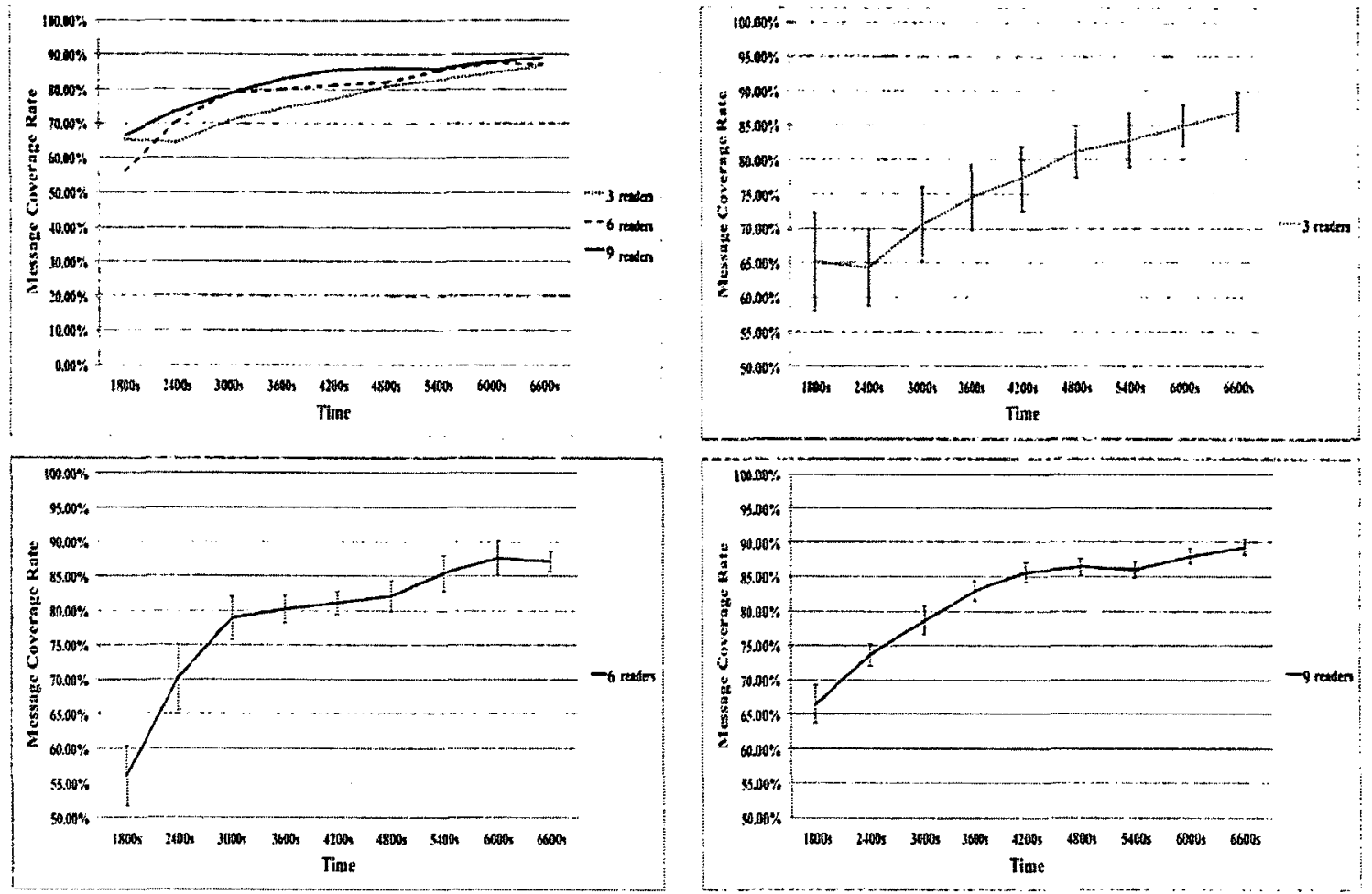

Figure 24: Message Coverage Rate with the $90 \%$ confidence interval.

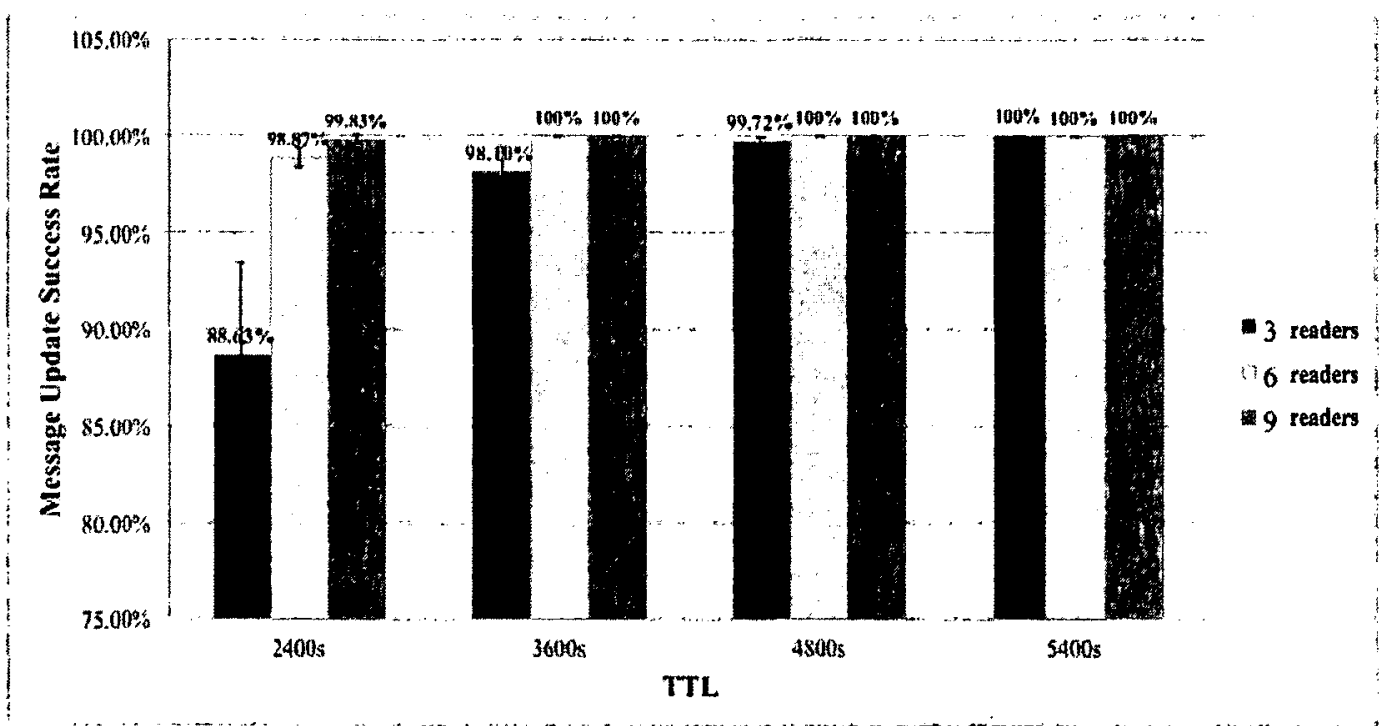

Figure 25: Message Update Success Rate with the $90 \%$ confidence interval. 


\subsection{Simulation conclusion}

Readers in the DTN based RFID protocol can connect with each other and exchange messages in a DTN region. By assigning proper TTLs, it is possible to ensure that messages reach and update all readers before they are dropped. From the simulation, we may conclude that the longer the TTL, the higher the possibility that a message can be received and updates reader local records before it is dropped. Also, the more readers in the DTN, the shorter TTL is needed to arrive at a $100 \%$ Message Update Success Rate. In case of a smaller size scenario map, the number of readers does not influence the result significantly. For a larger size scenario map, more readers in the DTN provide more connection possibilities and therefore a higher Message Coverage Rate is achieved. 


\section{Chapter 6}

\section{Conclusion}

In terms of ensuring the security of lightweight RFID tags, one of the difficulties is to meet security requirements within limited resources. Based on previous RFID studies, the DTN based RFID protocol has been proposed to ensure mutual verification, untraceability, forward security and backward security without permanent backend server connections. The ONE simulator has been used to evaluate the RFID reader performance in a DTN.

\subsection{The DTN based RFID protocol}

The DTN based RFID protocol enhances the security of the EPCglobal inventory protocol. Readers and tags share the same $P R N G$ state which includes the same $P R N G$ function and $P R N G$ seed to generate an identical number sequence for mutual verification. In Chapter 2, strategies are provided to resolve de-synchronizations and collisions. When a de-synchronization occurs, the reader and the tag are resynchronized before starting another inventory round for mutual verification. To solve a collision situation when several tags have the same TDS simultaneously, the reader requires one of the collided tags to temporally update its state and send new identification back. Then the reader is able to differentiate collided tags and to start a new inventory with the correct tag for mutual verification. 
A DTN is used to avoid permanent connections with a backend database or server. The $O N E$ simulator is used to evaluate the DTN's performance for RFID readers. The simulator $O N E$, illustrating a supermarket and a warehouse, shows how workers check and update product information by using readers. Reader holders walk randomly through the store and warehouse. A message is generated to update readers local records every time a holder stops and scans a product. When two readers are within each other's transmission range, new messages are broadcasted. Readers local records are updated by new messages and TTL expired messages are discarded. The simulation results imply that a proper TTL value is important for all readers to synchronize new messages and update their local records before messages are dropped.

\subsection{Performance comparison}

Table 8 compares performances of the Gossamer, Flyweight and DTN based RFID protocol.

\subsubsection{Computation cost}

The DTN based RFID protocol only uses the $X O R$ and $P R N G$ functions, which are compatible with the EPCglobal standard. The Gossamer protocol does not need to implement the $P R N G$ function to generate random numbers, but it does require tags to use new functions ROT and MIXBITS. As for the Flyweight protocol, the use of XOR operation and PRNG function are supported but they still require a new secure function to update the $P R N G$ seed for both a reader and a tag. 
Table 8: Performance comparison.

\begin{tabular}{l|lll}
\hline Performance & Gossamer & Flyweight & $\begin{array}{l}\text { DTN based RFID } \\
\text { protocol }\end{array}$ \\
\hline \hline Resynchronization & Yes & Yes & Yes \\
Data Confidentiality & Yes & Yes & Yes \\
Untraceability & Yes & Yes & Yes \\
Mutual Verification & Yes & Yes & Yes \\
Forward Security & Yes & Yes & Yes \\
Backward Security & NA & Yes & Yes \\
Communication Cost (bits) & 424 & 64 Or 80 & 66 \\
Memory Size on Tag (bits) & $21 * 32$ & $3 * 32+1$ & $2.5 * 32$ \\
$\begin{array}{l}\text { Memory Size for each Tag on } \\
\text { Reader/Database (bits) }\end{array}$ & $12 * 32$ & $6 * 32+1$ & $5 * 32$ \\
Functions and Operations & $\oplus,+, R O T$, & $\oplus, P R N G$, & $\oplus, P R N G$ \\
Permanent backend server & Yes & seed update function & \\
connections & & Yes & No \\
\hline \hline
\end{tabular}




\subsubsection{Communication cost}

For the cost of communication, the Gossamer protocol uses a total of 424 bits in four messages during the transmission for compatibility with the EPCglobal standard. The Flyweight protocol uses 64 bits in four messages in total when a tag and a reader are synchronized. If the tag and the reader are de-synchronized, then five messages are sent with a total of 80 bits. Moreover, another 32-bit random number is sent when the server updates a $P R N G$ seed. In the DTN based RFID protocol, four messages are transmitted, 66 bits in total.

\subsubsection{Storage cost}

To store all the necessary information of tags and readers, the Gossamer protocol requires a total of 1,056 bits for a tag along with its correlated information which is stored in the server while the flyweight protocol requires 290 bits. In the DTN based RFID protocol, a reader keeps 160 bits for one tag while a tag stores 80 bits locally and uses TID as TD. Thus, 240 bits in total. 


\section{List of References}

[1] Gildas Avoine, Xavier Carpent, and Benjamin Martin. Strong Authentication and Strong Integrity (SASI) is not that strong. In SiddikaBerna Ors Yalcin, editor, Radio Frequency Identification: Security and Privacy Issues, volume 6370 of Lecture Notes in Computer Science, pages 50-64. Springer Berlin Heidelberg, Jun. 2010.

[2] Michel Barbeau. Passive RFID tag security. In Haking pratical protection IT security magazine, volume 6, pages 30-37. Software Press Sp. z 0.0. SK 02-682 Warszawa, ul. Bokserska 1, Aug. 2011.

[3] Mike Burmester and Breno Medeiros. The Security of EPC Gen2 Compliant RFID Protocols. In StevenM. Bellovin, Rosario Gennaro, Angelos Keromytis, and Moti Yung, editors, Applied Cryptography and Network Security, volume 5037 of Lecture Notes in Computer Science, pages 490-506. Springer Berlin Heidelberg, 2008.

[4] Mike Burmester, Breno Medeiros, Jorge Munilla, and Alberto Peinado. Secure EPC Gen2 Compliant Radio Frequency Identification. In PedroM. Ruiz and JoseJoaquin Garcia-Luna-Aceves, editors, Ad-Hoc, Mobile and Wireless Networks, volume 5793 of Lecture Notes in Computer Science, pages 227-240. Springer Berlin Heidelberg, 2009.

[5] Mike Burmester and Jorge Munilla. A Flyweight RFID Authentication Protocol. In Workshop on RFID Security - RFIDSec'09, Leuven, Belgium, Jul. 2009.

[6] Chin-Ling Chen and Yong-Yuan Deng. Conformation of EPC Class 1 Generation 2 standards RFID system with mutual authentication and privacy protection. Engineering Applications of Artificial Intelligence, 22(8):1284 - 1291, Dec. 2009.

[7] Hung-Yu Chien. SASI: A New Ultralightweight RFID Authentication Protocol Providing Strong Authentication and Strong Integrity. Dependable and Secure Computing, IEEE Transactions on, 4(4):337 -340, Oct.-Dec. 2007. 
[8] Hung-Yu Chien and Che-Hao Chen. Mutual authentication protocol for RFID conforming to EPC Class 1 Generation 2 standards. Computer Standards Interfaces, 29(2):254 - 259, Feb. 2007.

[9] Don Coppersmith, Hugo Krawczyk, and Yishay Mansour. The Shrinking Generator. In Stinson, DouglasR., editor, Advances in Cryptology - CRYPTO' 93, volume 773 of Lecture Notes in Computer Science, pages 22-39. Springer Berlin Heidelberg, 1994.

[10] EPC Global. EPCglobal Architecture Framework. http://www .gs1.org/gsmp/kc/ epcglobal/architecture, 2010.

[11] EPC Global. Class 1 Generation 2 UHF Air Interface Protocol Standard. http: //www.gs1.org/gsmp/kc/epcglobal/uhfc1g2, 2011.

[12] EPC Global. EPC Radio-Frequency Identity Protocols EPC Class-1 HF RFID Air Interface Protocol. http://www .gs1 . org/gsmp/kc/epcglobal/hf, 2011.

[13] EPC Global. EPC Tag Data Standard (TDS). http://www.gs1.org/gsmp/kc/ epcglobal/tds/, 2011.

[14] EPC Global. EPC Tag Data Translation Standard (TDT). http://www.gs 1.org/ gsmp/kc/epcglobal/tdt/, 2011.

[15] EPC Global. EPCglobal Standards. http://www.gs1.org/gsmp/kc/epcglobal, 2011.

[16] Kevin Fall. A delay-tolerant network architecture for challenged internets. In Proceedings of the 2003 conference on Applications, technologies, architectures, and protocols for computer communications, SIGCOMM '03, pages 27-34, New York, NY, USA, 2003. ACM.

[17] Oded Goldreich, Shafi Goldwasser, and Silvio Micali. How to construct random functions. J. ACM, 33(4):792-807, Aug. 1986.

[18] John Kelsey, Bruce Schneier, David Wagner, and Chris Hall. Cryptanalytic Attacks on Pseudorandom Number Generators. In Vaudenay, Serge, editor, Fast Software Encryption, volume 1372 of Lecture Notes in Computer Science, pages 168-188. Springer Berlin Heidelberg, 1998.

[19] Ari Keränen, Jörg Ott, and Teemu Kärkkäinen. The ONE simulator for DTN protocol evaluation. In Proceedings of the 2nd International Conference on Simulation Tools 
and Techniques, Simutools '09, pages 55:1-55:10, ICST, Brussels, Belgium, Belgium, 2009. ICST (Institute for Computer Sciences, Social-Informatics and Telecommunications Engineering).

[20] H.R. Lee and D.W. Hong. The tag authentication scheme using self-shrinking generator on RFID system. Transactions on Engineering, Computing, and Technology, 18:52-57, Dec. 2006.

[21] Willi Meier and Othmar Staffelbach. The self-shrinking generator. In Alfredo Santis, editor, Advances in Cryptology - EUROCRYPT'94, volume 950 of Lecture Notes in Computer Science, pages 205-214. Springer Berlin Heidelberg, 1995.

[22] Pedro Peris-Lopez, Julio C. Hernandez-Castro, JuanM Estevez-Tapiador, and Jan C.A. van der Lubbe. Cryptanalysis of an EPC class-1 generation-2 standard compliant authentication protocol. Engineering Applications of Artificial Intelligence, 24(6): 1061-1069, Sep. 2011.

[23] Pedro Peris-Lopez, Julio Cesar Hernandez-Castro, Juan M. Estevez-Tapiador, and Arturo Ribagorda. LAMED - A PRNG for EPC Class-1 Generation-2 RFID specification. Comput. Stand. Interfaces, 31(1):88-97, Jan. 2009.

[24] Pedro Peris-Lopez, JulioCesar Hernandez-Castro, JuanM. Estevez-Tapiador, and Arturo Ribagorda. EMAP: An efficient mutual-authentication protocol for low-cost RFID tags. In Robert Meersman, Zahir Tari, and Pilar Herrero, editors, On the Move to Meaningful Internet Systems 2006: OTM 2006 Workshops, volume 4277 of Lecture Notes in Computer Science, pages 352-361. Springer Berlin Heidelberg, 2006.

[25] Pedro Peris-Lopez, JulioCesar Hernandez-Castro, JuanM. Estevez-Tapiador, and Arturo Ribagorda. LMAP: A real lightweight mutual authentication protocol for lowcost RFID tags. In In: Proc. of 2nd Workshop on RFID Security, page 06. Ecrypt, Jul. 2006.

[26] Pedro Peris-Lopez, JulioCesar Hernandez-Castro, JuanM. Estevez-Tapiador, and Arturo Ribagorda. $\mathrm{M}^{2} \mathrm{AP}$ : a minimalist mutual-authentication protocol for low-cost RFID tags. In Jianhua Ma, Hai Jin, LaurenceT. Yang, and JeffreyJ.-P. Tsai, editors, Ubiquitous Intelligence and Computing, volume 4159 of Lecture Notes in Computer Science, pages 912-923. Springer Berlin Heidelberg, 2006.

[27] Pedro Peris-Lopez, JulioCesar Hernandez-Castro, JuanM. Estevez-Tapiador, and Arturo Ribagorda. Cryptanalysis of a novel authentication protocol conforming to EPCC1G2 standard. Computer Standards Interfaces, 31(2):372 - 380, Feb. 2009. 
[28] Pedro Peris-Lopez, JulioCesar Hernandez-Castro, JuanM.E. Tapiador, and Arturo Ribagorda. Advances in ultralightweight cryptography for low-cost RFID tags: Gossamer protocol. In Kyo-Il Chung, Kiwook Sohn, and Moti Yung, editors, Information Security Applications, volume 5379 of Lecture Notes in Computer Science, pages 56-68. Springer Berlin Heidelberg, 2009.

[29] R.C.-W. Phan. Cryptanalysis of a New Ultralightweight RFID Authentication Protocol x02014;SASI. Dependable and Secure Computing, IEEE Transactions on, 6(4):316 -320, Oct.-Dec. 2009.

[30] Thrasyvoulos Spyropoulos, Konstantinos Psounis, and Cauligi S. Raghavendra. Spray and wait: an efficient routing scheme for intermittently connected mobile networks. In Proceedings of the 2005 ACM SIGCOMM workshop on Delay-tolerant networking, WDTN '05, pages 252-259, New York, NY, USA, 2005. ACM.

[31] A. Vahdat, D. Becker, et al. Epidemic routing for partially connected ad hoc networks. Technical report, Technical Report CS-2000-06, Duke University, 2000. 